\title{
Neurotransmitters and Motoneuron Contacts of Multifunctional and Behaviorally Specialized Turtle Spinal Cord Interneurons
}

\author{
B. Anne Bannatyne, ${ }^{1}$ Zhao-Zhe Hao (郝赵哲), ${ }^{2}$ Georgia M. C. Dyer, ${ }^{1}$ CMasahiko Watanabe, ${ }^{3}$ David J. Maxwell, ${ }^{1}$ \\ and $\odot$ Ari Berkowitz ${ }^{2}$ \\ ${ }^{1}$ Spinal Cord Group, Institute of Neuroscience and Psychology, University of Glasgow, United Kingdom G12 8QQ, ${ }^{2}$ Department of Biology and Cellular and \\ Behavioral Neurobiology Graduate Program, University of Oklahoma, Norman, Oklahoma 73019, and ${ }^{3}$ Department of Anatomy, Hokkaido University \\ Faculty of Medicine, Sapporo 060-8638, Japan
}

The spinal cord can appropriately generate diverse movements, even without brain input and movement-related sensory feedback, using a combination of multifunctional and behaviorally specialized interneurons. The adult turtle spinal cord can generate motor patterns underlying forward swimming, three forms of scratching, and limb withdrawal (flexion reflex). We previously described turtle spinal interneurons activated during both scratching and swimming (multifunctional interneurons), interneurons activated during scratching but not swimming (scratch-specialized interneurons), and interneurons activated during flexion reflex but not scratching or swimming (flexion reflex-selective interneurons). How multifunctional and behaviorally specialized turtle spinal interneurons affect downstream neurons was unknown. Here, we recorded intracellularly from spinal interneurons activated during these motor patterns in turtles of both sexes in vivo and filled each with dyes. We labeled motoneurons using choline acetyltransferase antibodies or earlier intraperitoneal FluoroGold injection and used immunocytochemistry of interneuron axon terminals to identify their neurotransmitter(s) and putative synaptic contacts with motoneurons. We found that multifunctional interneurons are heterogeneous with respect to neurotransmitter, with some glutamatergic and others GABAergic or glycinergic, and can directly contact motoneurons. Also, scratch-specialized interneurons are heterogeneous with respect to neurotransmitter and some directly contact motoneurons. Thus, scratch-specialized interneurons might directly excite motoneurons that are more strongly activated during scratching than forward swimming, such as hip-flexor motoneurons. Finally, and surprisingly, we found that some motoneurons are behaviorally specialized, for scratching or flexion reflex. Thus, either some limb muscles are only used for a subset of limb behaviors or some limb motoneurons are only recruited during certain limb behaviors.

Key words: central pattern generation; flexion reflex; limb withdrawal; locomotion; scratching; swimming

Significance Statement

Both multifunctional and behaviorally specialized spinal cord interneurons have been described in turtles, but their outputs are unknown. We studied responses of multifunctional interneurons (activated during swimming and scratching) and scratchspecialized interneurons, filled each with dyes, and used immunocytochemistry to determine their neurotransmitters and contacts with motoneurons. We found that both multifunctional and scratch-specialized interneurons are heterogeneous with respect to neurotransmitter, with some excitatory and others inhibitory. We found that some multifunctional and some scratchspecialized interneurons directly contact motoneurons. Scratch-specialized interneurons may excite motoneurons that are more strongly activated during scratching than swimming, such as hip-flexor motoneurons, or inhibit their antagonists, hip-extensor motoneurons. Surprisingly, we also found that some motoneurons are behaviorally specialized, for scratching or for flexion reflex.

\section{Introduction}

Animals use many of the same muscles to generate a wide variety of movements. How the nervous system coordinates the timing

Received Sept. 11, 2019; revised Jan. 31, 2020; accepted Feb. 6, 2020

Author contributions: B.A.B. designed research; B.A.B., Z.-Z.H., and A.B. performed research; M.W. contributed unpublished reagents/analytic tools; B.A.B., G.M.C.D., D.J.M., and A.B. analyzed data; B.A.B., D.J.M., and A.B. wrote the paper. and amplitude of muscle contractions to control each behavior appropriately is best understood for certain invertebrates in which most of the neurons involved contribute to generating 
multiple behaviors (i.e., they are multifunctional neurons), but some neurons are instead dedicated or behaviorally specialized (Morton and Chiel, 1994; Briggman and Kristan, 2008). Among vertebrates, the control of axial movements in very young animals is best understood, especially for swimming versus struggling in hatchling tadpoles and larval zebrafish (Berkowitz et al., 2010). In these cases, also, a combination of multifunctional and behaviorally specialized spinal cord interneurons coordinates motor output.

How distinct limb behaviors are selected and generated in adult vertebrates is of great interest but is poorly understood, due mainly to the larger number of neurons involved and technical obstacles. The adult turtle spinal cord can generate the motor patterns underlying forward swimming, three forms of scratching, and limb withdrawal (flexion reflex), even in the absence of brain inputs and movement-related sensory feedback (Stein, 2005). Thus, the organization of the spinal cord network(s) that control these behaviors can conveniently be studied in turtles, which also have the advantage of a resistance to hypoxia that is unusual among adult vertebrates (Hounsgaard and Nicholson, 1990; Lutz and Milton, 2004).

We previously found that many turtle spinal interneurons activated during scratching motor patterns are also activated during swimming (scratch/swim or multifunctional interneurons) and often during flexion reflex as well (Berkowitz, 2002, 2008, 2010). However, we also found that there are behaviorally specialized spinal interneurons activated during scratching but not swimming (scratch-specialized interneurons; Berkowitz, 2002, 2008,2010 ) and others activated during flexion reflex but not scratching or swimming (flexion reflex-selective interneurons; Berkowitz, 2007, 2010). These previous studies addressed the circumstances under which multifunctional and behaviorally specialized interneurons are activated (i.e., their inputs), but not their outputs. To better understand how a combination of multifunctional and behaviorally specialized interneurons generates the right movement at the right time in adult limbed vertebrates, it is necessary to determine their outputs as well, including whether they are excitatory or inhibitory and whether they contact motoneurons directly.

Such information can provide a foundation for constructing hypotheses and for comparison to species in which multifunctional and behaviorally specialized interneurons have been elucidated in more detail, especially hatchling Xenopus tadpoles and larval zebrafish (Berkowitz et al., 2010). The turtle spinal cord has been a focus of research on spinal control of multiple kinds of natural limb movements for $>4$ decades (Lennard and Stein, 1977; Valk-Fai and Crowe, 1978; Stein, 2018) and has also been a focus for investigation of diverse cellular- and circuit-level questions of general interest, including the ion channel mechanisms and modulation of plateau potentials, windup, and long-lasting excitability (Hounsgaard and Kiehn, 1989; Currie and Stein, 1990; Hounsgaard and Kjaerulff, 1992; Russo and Hounsgaard, 1994; Delgado-Lezama et al., 1997, 1999; Russo et al., 1997; Guertin and Hounsgaard, 1998; Svirskis and Hounsgaard, 1998; Alaburda and Hounsgaard, 2003; Alaburda et al., 2005; Guzulaitis et al., 2013; Reali and Russo, 2013; Johnson et al., 2017), the contribution of rhythmic inhibition to spinal central patterngenerating networks (CPGs; Robertson and Stein, 1988;

The authors declare no competing financial interests.

Correspondence should be addressed to Ari Berkowitz at ari@ou.edu.

https://doi.org/10.1523/JNEUROSCI.2200-19.2020

Copyright $(2020$ the authors
Berkowitz and Stein, 1994b; Berg et al., 2007; Berkowitz, 2008; Stein, 2010; Petersen et al., 2014; Guzulaitis and Hounsgaard, 2017), the distribution, sparseness, and modularity of spinal cord CPGs (Mortin and Stein, 1989; Stein et al., 1995, 1998; Currie and Gonsalves, 1997; Stein, 2008; Guzulaitis et al., 2014; Hao et al., 2014; Radosevic et al., 2019), and interactions of spinal CPGs (Stein et al., 1986; Currie and Stein, 1989; Earhart and Stein, 2000; Hao et al., 2011; Elson and Berkowitz, 2016; Hao and Berkowitz, 2017). Therefore, it is important to gather foundational data on the neurotransmitters and synaptic contacts of functional interneuron types in this system.

Here, we used dye-filled sharp microelectrodes to record intracellularly from spinal neurons while eliciting motor patterns for forward swimming (henceforth, just "swimming"), scratching, and flexion reflex in immobilized, spinally transected, adult turtles, then used current injection to fill each characterized neuron with dyes. We then used immunocytochemistry on dye-filled axon terminals to identify each interneuron's neurotransmitter phenotype and to look for synaptic contacts between interneuron axon terminals and motoneuron somata/dendrites. We found that multifunctional interneurons can be either excitatory (glutamatergic) or inhibitory (GABAergic or glycinergic). Some multifunctional interneurons contact motoneurons directly. We found that scratch-specialized interneurons are heterogeneous with respect to neurotransmitter and some contact motoneurons directly. Finally, we found, surprisingly, that motoneurons can be behaviorally specialized as well. Some of these findings have been reported previously in an abstract (Berkowitz et al., 2018).

\section{Materials and Methods}

Surgical procedures. Adult red-eared turtles, Trachemys scripta elegans, of both sexes $(n=34)$, weighing 300-1140 g, were purchased from Charles Sullivan, Kons Direct, or Cyr's Biology. Animals were injected with FluoroGold ( $200 \mu$ l, 1\%, i.p.; Fluorochrome) 1-2 months before the experiment to retrogradely label motoneurons (Ambalavanar and Morris, 1989). Surgical procedures were described previously (Robertson et al., 1985; Berkowitz, 2001a) and are summarized briefly here. Dissection was performed under hypothermic analgesia to (1) transect the spinal cord between the dorsal 2 (D2) and D3 postcervical segments, (2) expose the spinal cord between the D6 and sacral 2 (S2) segments, and (3) prepare several right hindlimb motor nerves for extracellular recordings and stimulation: the hip flexor (HF), ventral puboischiofemoralis internus, pars anteroventralis; the hip extensor (HE), flexor cruris, pars flexor tibialis internus; and the knee extensors, triceps femoralis, pars iliotibialis, pars ambiens, and/or pars femorotibialis (Robertson et al., 1985), and in some experiments, the distal D8 (dD8) segmental nerve (Mortin and Stein, 1989). After surgery, turtles were warmed to room temperature for $0.5 \mathrm{~h}$, immobilized with gallamine triethiodide $(8 \mathrm{mg} / \mathrm{kg}$, i.m.; SigmaAldrich), and artificially ventilated throughout the experiment. All animal procedures were approved by the Institutional Animal Care and Use Committee of the University of Oklahoma.

Stimulation procedures. Forward-swimming motor patterns were evoked by electrical stimulation (Master-8 stimulator with ISO-Flex stimulus isolation unit, AMPI) in the D3 contralateral lateral funiculus $(0.1 \mathrm{~ms}, 200-400 \mu \mathrm{A}$, bipolar pulses at $40 \mathrm{~Hz})$ via a pair of $100 \mu \mathrm{m}$ silver wires (California Fine Wire), insulated except at the tips, with one tip contacting the $\mathrm{D} 3$ face of the spinal cord and the other in the surrounding saline (Lennard and Stein, 1977; Juranek and Currie, 2000; Berkowitz, 2002). Rostral, pocket, and caudal scratching motor patterns were evoked by continual gentle rubbing of a single site in the receptive field of each scratch form at $\sim 3 \mathrm{~N}, \sim 3-4 \mathrm{~Hz}$ using a glass probe with a firepolished tip (Mortin et al., 1985; Hao et al., 2014). Flexion reflex motor patterns were evoked either with a single tap to the dorsal foot using a glass probe with a fire-polished tip (Currie and Lee, 1996) or with a train of five electrical pulses ( $1 \mathrm{~ms}$ each at $100 \mathrm{~Hz}$ ) delivered via a pair of gold/silver cup electrodes with centers $\sim 1 \mathrm{~cm}$ apart attached to the dorsal 
foot skin with electrode cream (Grass/Astromed/Natus Neurology electrodes and cream; Master-8 stimulator, AMPI; Johnson et al., 2017).

Electrophysiology. Dissected nerves were submerged in mineral oil, surrounded by a wax well molded onto the turtle carapace. Recordings from each nerve were obtained using a pair of $100 \mu \mathrm{m}$ silver wires (California Fine Wire) and amplified and filtered $(\times 1000$; bandpass $0.1-1.0 \mathrm{kHz}$; A-M Systems or Natus Neurology amplifiers). Intracellular recordings were obtained with sharp microelectrodes in the ipsilateral D7-D10 and sacral 1-2 spinal segments, with the meninges torn at the site of each penetration. Intracellular microelectrodes were made using a $\mathrm{P}-97$ puller (Sutter Instrument), from $1.0 \mathrm{~mm}$ outer diameter $/ 0.78 \mathrm{~mm}$ inner diameter borosilicate glass with filament (Sutter Instrument). Microelectrodes were filled with 2.5\% 3000-MW tetramethylrhodamine dextran (ThermoFisher Scientific) and 2.5\% Neurobiotin (NB; Vector Laboratories) in either $0.5 \mathrm{M}$ potassium chloride (ThermoFisher Scientific) or $5 \%$ $\mathrm{NaCl}$ (ThermoFisher Scientific), pH 7.0. In most cases, microelectrodes were slightly beveled (BV-10, Sutter Instrument). Microelectrodes had electrical resistances of 30-120 M $\Omega$. Intracellular recordings were obtained with an Intra 767 amplifier (World Precision Instruments). The microelectrode was advanced using a Burleigh Inchworm piezoelectric microdrive (Burleigh/EXFO). Both nerve and single-cell recordings were stored on a digital audio tape recorder (TEAC America) and on a PC (Micro1401 hardware and Spike2 software, Cambridge Electronic Design). Dyes were ejected via net-positive $4 \mathrm{~Hz}$ sinusoidal current injection (1.6-4.0 nA for 5-50 min; AFG-2021 function generator, Tektronix; Intra 767 amplifier, World Precision Instruments).

Histology. Following the last attempted dye injection of a characterized cell, the animal was deeply anesthetized (Euthanasia III; Western Medical Supply or Patterson Veterinary Supply; $390 \mathrm{mg} / \mathrm{ml}, 1.0 \mathrm{ml}$, i.p.). One hour after the anesthetic injection, the heart was exposed and perfused with $40-80 \mathrm{ml}$ turtle saline containing $0.1 \%$ sodium nitrite (SigmaAldrich), $10 \mathrm{U} / \mathrm{ml}$ heparin (Sigma-Aldrich), and $0.1 \mathrm{ml}$ Euthanasia III, followed by $300 \mathrm{ml}$ cold $4 \%$ paraformaldehyde (Sigma-Aldrich) in $0.1 \mathrm{M}$ phosphate buffer (ThermoFisher Scientific), pH 7.4 (PB). The D7-S2 spinal cord was then removed and pinned out straight in a Sylgard-lined (Dow Corning) Petri dish and submerged in the same fixative solution at $4^{\circ} \mathrm{C}$ overnight. The spinal cord was then transferred to a tube containing $30 \%$ sucrose and $0.1 \%$ paraformaldehyde in $\mathrm{PB}$ at $4^{\circ} \mathrm{C}$ and later shipped on an ice pack from the University of Oklahoma to the University of Glasgow via 2-d courier.

Immunocytochemistry. Dorsal rootlets were identified to determine segmental boundaries and the spinal cord was cut into blocks. Transverse sections ( $50 \mu \mathrm{m}$ thick) were cut by using a Vibratome VT $1000 \mathrm{~S}$ (Leica Biosystems) and collected in strict serial order. Sections were rinsed for $30 \mathrm{~min}$ in $50 \%$ ethanol to enhance antibody penetration and, following several washes in PBS, were mounted in serial order with VECTASHIELD (Vector Laboratories) on glass slides with coverslips. Sections were examined with a fluorescence microscope and series of sections containing cells labeled with tetramethylrhodamine dextran were selected and incubated with avidin-rhodamine for $3 \mathrm{~h}$ (1:1000, Jackson ImmunoResearch) to reveal the full extent of the NB labeling. Sections were mounted for a second time and cells were scanned with a confocal microscope (Zeiss LSM 710, Carl Zeiss) to enable reconstructions of axonal and dendritic arbors to be made ( $20 \times$ lens). Sections containing cells with axonal projections and terminal-like swellings were removed from slides and prepared for immunocytochemistry (Table 1). The following two antibody combinations were used: (1) anti-gephyrin, which labels the postsynaptic densities of inhibitory synapses (Triller et al., 1985; Essrich et al., 1998); anti-Homer, which labels the postsynaptic densities of excitatory synapses (Gutierrez-Mecinas et al., 2016); anticholine acetyltransferase (ChAT; to label motoneurons; Lee et al., 2007; Radosevic et al., 2019); or (2) anti-vesicular glutamate transporter 2 (VGLUT2; to label excitatory terminals); anti-glutamic acid decarboxylase (GAD) or anti-glycine transporter2 (GlyT2; to label inhibitory terminals). In addition, sections containing labeled motoneurons were incubated with anti-ChAT and anti-FluoroGold (FG). All antibodies were diluted in Tris PBS and sections were incubated for $72 \mathrm{~h}$. They were rinsed in PBS and incubated for $3 \mathrm{~h}$ in solutions of donkey secondary antibodies coupled to fluorophores (Jackson ImmunoResearch; Table 1)
Table 1. Antibody combinations used in the study

\begin{tabular}{lllll}
\hline Section content & $\begin{array}{l}\text { Primary antibody } \\
\text { combination }\end{array}$ & $\begin{array}{l}\text { Primary antibody } \\
\text { concentration }\end{array}$ & Source & $\begin{array}{l}\text { Secondary } \\
\text { antibodies }\end{array}$ \\
\hline Axon terminals & Rabbit anti-Homer & $1: 500$ & M. Watanabe & AlexaFluor 488 \\
& Mouse anti-gephyrin & $1: 500$ & Synaptic Systems & AlexaFluor 647 \\
& Goat anti-ChAT & $1: 100$ & Millipore & Pacific Blue \\
Axon terminals & Guinea pig anti-VGLUT2 & $1: 5000$ & Millipore & AlexaFluor 647 \\
& Rabbit anti-GAD OR & $1: 1000$ & Sigma-Aldrich & AlexaFluor 488 \\
& Sheep anti-GlyT2 & $1: 1000$ & Chemicon & AlexaFluor 488 \\
& Goat anti-ChAT & $1: 100$ & Millipore & Pacific Blue \\
Motoneurons & Goat anti-ChAT & $1: 100$ & Millipore & AlexaFluor 647 \\
& Rabbit anti-FG & $1: 2000$ & Millipore & Pacific Blue \\
\hline
\end{tabular}

and, following several further washes in PBS were mounted on glass slides for a third time with VECTASHIELD. Each section was examined systematically with the confocal microscope at a high magnification by using a $63 \times$ oil-immersion lens at zoom factor of 2 . Series of four-color confocal images were gathered at intervals of $0.3 \mu \mathrm{m}$ to determine the neurotransmitter type associated with each cell and if contacts were made with motoneurons.

Antibody specificity. Most of the antibodies used in this study have been used extensively in investigations of the mammalian nervous system and, in some cases, also for non-mammalian species. We initially tested a large range of primary antibodies on turtle spinal tissue and selected only those antibodies that produced an identical staining pattern in mammalian studies. We also conducted a series of positive control experiments which confirmed that the antibodies were binding to their predicted targets. The rabbit anti-Homer antibody is new, so we tested it against a goat anti-Homer antibody. The two antibodies labeled the same structures in individual turtle spinal sections.

Neuronal reconstruction. In total, 37 interneurons were reconstructed three-dimensionally by using Neurolucida for confocal software (MicroBrightField). Reconstructions were made from tiled stacks of single optical sections. The contours of the gray matter and central canal were added to provide landmarks to determine the locations of cells and their processes within the structures of the spinal cord. Cell body locations of all 37 cells were plotted on a schematized template of a transverse spinal section.

The Neurolucida program also provided quantitative data about dendritic length and orientation. Transverse interneurons (T neurons) were identified and classified as described by Berkowitz et al. (2006) using ratios of somatic and dendritic length measurements extracted from Neurolucida reconstructions. The dendritic ratio was calculated as [dendritic rostrocaudal $/($ mediolateral + dorsoventral $)<0.4$; i.e., $\mathrm{RC} /(\mathrm{ML}+$ $\mathrm{DV})<0.4$ ] and the somatic ratio as [somatic RC/ML $<1)$. Cells meeting both criteria were termed $\mathrm{T}(1)$ neurons and cells meeting only the dendritic criterion were termed $\mathrm{T}(2)$ neurons (Berkowitz, 2008). Non-T neurons were defined as cells with dendritic $\mathrm{RC} /(\mathrm{ML}+\mathrm{DV})>0.4$.

The use of gephyrin and Homer in these studies enabled us to determine whether interneuron terminals formed synaptic associations with motoneurons. The relationship between an individual NB-labeled terminal and a postsynaptic density marker was always examined through series of thin optical sections and multiple terminals from each cell were examined to confirm that the pattern of apposition was consistent for all terminals.

Electrophysiological data analysis. Recordings of interneurons were analyzed whenever the soma was unambiguously recovered histologically and confirmed to be that of an interneuron, due to lack of FluoroGold and anti-ChAT staining. In general, recordings of motoneurons were not analyzed, but two confirmed motoneurons are shown (see Figs. 10, 11) and described because of their surprising behavioral specialization. The action potential width of each intracellularly recorded interneuron was measured at the action potential threshold when the neuron was firing at a low rate. Dual-referent phase histograms (Berkowitz and Stein, 1994b) of intracellularly recorded neurons were calculated with respect to the onset and offset of bursts of a nerve, usually HF but in some cases HE or dD8 instead, during scratching and/or swimming motor patterns, using a custom script written in Spike2 (Cambridge Electronic Design). 


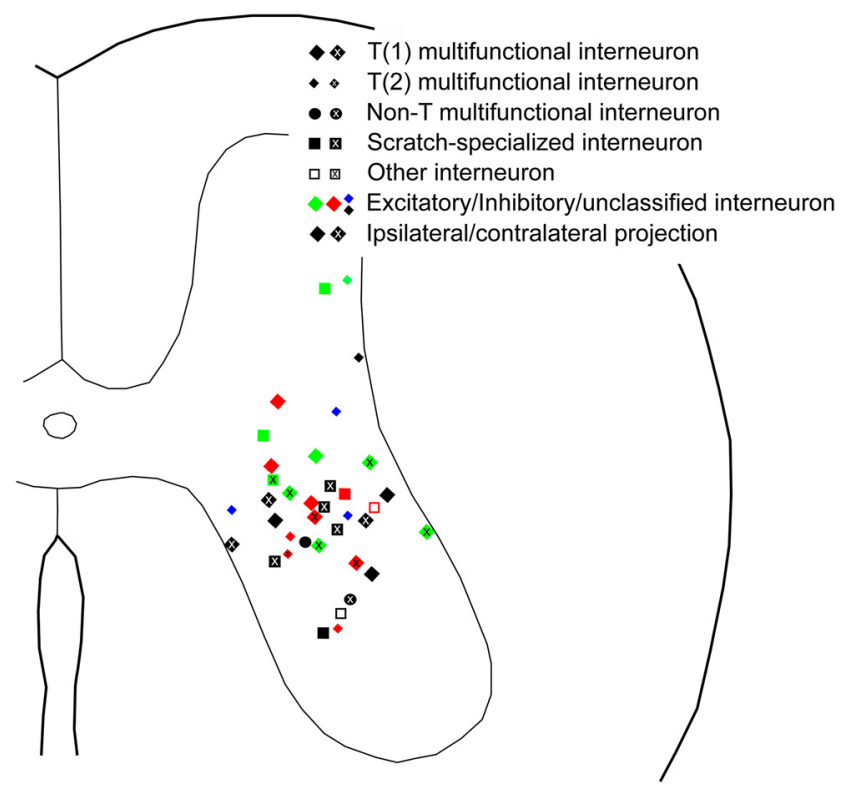

Figure 1. Soma locations of all intracellularly recorded and dye-filled interneurons unambiguously recovered, categorized by functional and morphological characteristics.

The peak-trough amplitude and peak phase of intracellular membrane potential oscillations were calculated after deleting action potentials and interpolating voltage values between (Berkowitz, 2005). If the peak phase was calculated with respect to HE, then 0.5 was added or subtracted to the peak phase to assess all peak phases with respect to the HF activity cycle. The peak phase and peak amplitude reported for each neuron are those for the rhythmic motor pattern (swimming or a form of scratching) that had the largest membrane potential oscillation amplitude. Mean amplitudes of hip-flexor nerve bursts were measured in Spike2 after DC-level correction, rectification, and smoothing (100 ms sliding window). Spiketriggered averaging of hip-flexor nerve bursts was performed in Spike 2.

Experimental design and statistical analysis. The Mann-Whitney test (InStat, GraphPad Software) was used for statistical comparisons of linear parameters and the Rayleigh test for statistical comparisons of phase values.

\section{Results}

Thirty-seven interneurons were characterized physiologically, filled with dyes, and the soma unambiguously recovered histologically (Fig. 1). Of these, 22 were activated during both swimming and scratching motor patterns (multifunctional interneurons); 13 of these 22 were also activated during flexion reflex motor patterns and 2 were not tested during flexion reflex. Six of the 37 interneurons were activated during scratching but not tested during swimming, because swimming was not successfully evoked in those animals; 3 of those 6 interneurons were also activated during flexion reflex. Nine of the 37 interneurons were activated during scratching but not swimming (scratch-specialized interneurons); 5 of these 9 were also activated during flexion reflex and one was not tested. Thus, for the 29 interneurons tested during swimming, scratching, and flexion reflex, 13 (45\%) were activated during all 3 kinds of motor patterns, 8 (28\%) were activated during swimming and scratching but not flexion reflex, $5(17 \%)$ were activated during scratching and flexion reflex but not swimming, and the remaining 3 (10\%) were activated during scratching but not swimming or flexion reflex.

There were no consistent differences between multifunctional and scratch-specialized interneurons with respect to action potential width ( $p=0.20$, Mann-Whitney test) or membrane potential oscillation amplitude during swimming/scratching
( $p=0.27$, Mann-Whitney test). The action potential widths (mean $\pm \mathrm{SD}$ ) were $2.6 \pm 1.7 \mathrm{~ms}$ for multifunctional interneurons and $1.8 \pm 0.7 \mathrm{~ms}$ for scratch-specialized interneurons. The membrane potential oscillation amplitudes were $5.0 \pm 3.3 \mathrm{mV}$ for multifunctional interneurons and $7.0 \pm 4.4 \mathrm{mV}$ for scratchspecialized interneurons. The largest oscillation amplitude was during a form of scratching for 19 of the multifunctional interneurons and during swimming for the remaining three multifunctional interneurons. The peak depolarizing phase (see Materials and Methods) was during the HF bursts (0.0-0.49 phase) for 6 of the multifunctional interneurons (individual values of $0.00,0.30,0.33,0.33,0.38$, and 0.40 ) and between the HF bursts (0.50-0.99 phase) for the remaining 16 multifunctional interneurons (individual values of $0.50,0.50,0.50,0.54,0.57$, $0.68,0.72,0.79,0.79,0.81,0.86,0.88,0.93,0.94,0.94$, and 0.95 ). The peak depolarizing phase was during the HF bursts for 5 of the scratch-specialized interneurons (individual values of $0.00,0.01$, $0.03,0.16$, and 0.36 ) and between the HF bursts for the remaining 4 scratch-specialized interneurons (individual values of 0.51 , $0.68,0.68$, and 0.99 ). These peak depolarizing phases were not significantly different from random distributions for either multifunctional interneurons ( $r=0.21 ; p=0.32$; Rayleigh test) or scratch-specialized interneurons $(r=0.23 ; p=0.61$; Rayleigh test).

Of the 37 interneurons characterized physiologically with soma recovered unambiguously, NB-labeled axon terminals connected to the soma were successfully reacted with antibodies to determine the neurotransmitter phenotype for 13 multifunctional interneurons ( 7 having ipsilateral axons, 6 having contralateral axons), 2 scratch-activated interneurons for which swimming could not be tested (1 having an ipsilateral axon and 1 a contralateral axon), and 4 scratch-specialized interneurons ( 3 having an ipsilateral axon and 1 a contralateral axon). Only one of these interneurons had a bilaterally projecting axon.

\section{Neurotransmitters of multifunctional interneurons}

We determined the neurotransmitter(s) used by each interneuron using antibodies to the (postsynaptic) Homer (Ho) protein or the (presynaptic) vesicular glutamate transporter 2 to identify glutamatergic neurons, antibodies to the (postsynaptic) gephyrin protein ("7a") to identify GABAergic/glycinergic neurons, and antibodies to (presynaptic) ChAT to identify cholinergic neurons.

Reconstructions of all multifunctional interneurons with successfully reacted terminals are shown in Figures 2 and 3. We found that our sample of multifunctional interneurons was heterogeneous with respect to neurotransmitter, including both excitatory and inhibitory interneurons. Most excitatory multifunctional interneurons ( 3 of 5; Fig. 2) and inhibitory multifunctional interneurons ( 6 of 8 ; Fig. 3 ) had axon terminals in the vicinity of the motor nuclei, ipsilaterally (Figs. $2 A, 3 A, B, D-F$ ) or contralaterally (Figs. 2C,E, 3G).

The multifunctional interneuron shown in Figure $4 A-C$ was rhythmically activated during rostral and caudal scratching and also activated during swimming and flexion reflex $(4 A)$. Its soma was in the dorsomedial part of the D8 ventral horn (Fig. $4 B$ ). It had axon terminals in the ipsilateral ventral horn and ventral funiculus (Figs. $4 B, C, 3 C$ ). Individual terminals of this interneuron (Fig. $4 C$, red) were closely apposed to punctate gephyrin antibody labeling (white) and not Homer antibody labeling (green), demonstrating that this interneuron was GABAergic or glycinergic. The multifunctional interneuron shown in Figure 4, $D$ and $E$, had its soma in the $S 1$ ventral horn and axon terminals in 

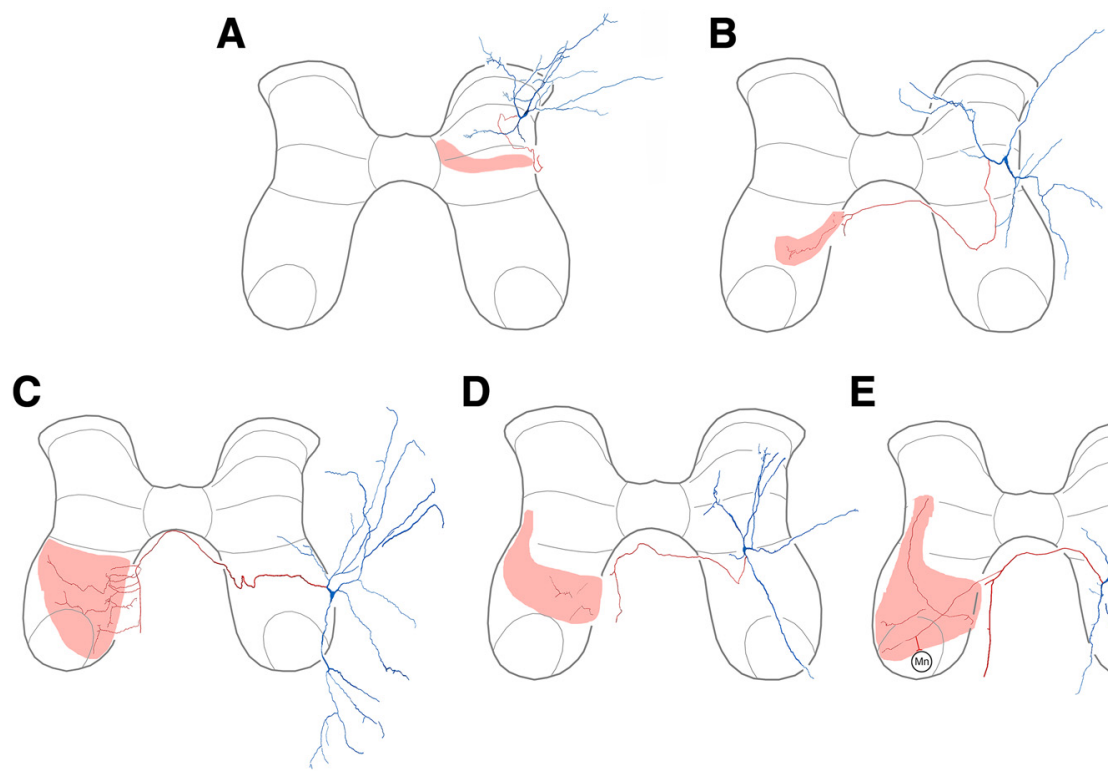

E

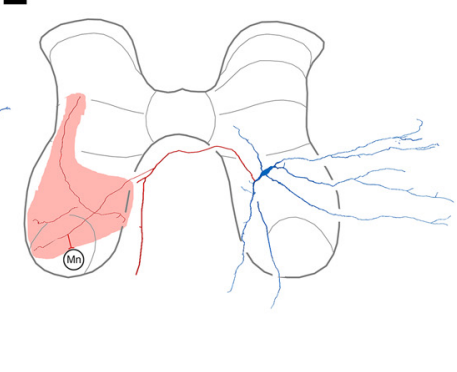

Figure 2. Reconstructions of excitatory multifunctional interneurons. $\boldsymbol{A}$, Interneuron with ipsilateral axon terminals. $\boldsymbol{B}-\boldsymbol{E}$, Interneurons with contralateral axon terminals. In each case, soma and dendrites are blue; axon and terminals are red; termination zone is pink.

the contralateral ventral horn (Figs. 4D, 2E). This interneuron's terminals (red) were closely apposed to punctate Homer antibody labeling (green), demonstrating that this interneuron was glutamatergic (Fig. 4E). The multifunctional interneuron shown in Figure 4, $F$ and $G$, had its soma in the D9 ventrolateral dorsal horn (Fig. $4 F$ ). It had axon terminals (red) in the ipsilateral dorsal horn (Figs. $4 F, 2 A$ ) that were closely apposed to vesicular glutamate transporter 2 antibody labeling (blue), demonstrating that this interneuron was also glutamatergic $(4 G)$.

Of the 13 multifunctional interneurons with identified axon terminals that were successfully reacted with antibodies, eight interneurons were GABAergic or glycinergic and thus likely inhibitory and five interneurons were glutamatergic and thus likely excitatory. The action potential widths were $3.4 \pm 2.0 \mathrm{~ms}$ for the inhibitory multifunctional interneurons and $2.7 \pm 2.1 \mathrm{~ms}$ for the excitatory multifunctional interneurons, which were not significantly different ( $p>0.99$, Mann-Whitney test). The oscillation amplitudes were $4.6 \pm 2.4 \mathrm{mV}$ for the inhibitory multifunctional interneurons and $7.1 \pm 5.0 \mathrm{mV}$ for the excitatory multifunctional interneurons, which were not significantly different $(p=0.42$, Mann-Whitney test). Peak depolarization occurred during the HF bursts for three of the inhibitory multifunctional interneurons (phases of 0.0, 0.38, and 0.40) and between the HF bursts for the remaining 5 (phases of $0.50,0.54,0.57,0.68$, and 0.95 ); this distribution was not significantly different from random $(p=$ 0.31 , Rayleigh test). Peak depolarization occurred during the HF bursts for one of the five excitatory multifunctional interneurons (phase of 0.30) and between the HF bursts for the remaining four (phases of $0.72,0.79,0.86$, and 0.94 ); this distribution was not significantly different from random ( $p=0.34$, Rayleigh test).

We previously described a morphologically defined category of scratch-activated interneurons, called transverse interneurons (T neurons), having dendrites that extended far within the transverse plane but were relatively short rostrocaudally; they also had mediolaterally elongated somata (Berkowitz et al., 2006) and their dendrites tended to branch less and be angled more mediolaterally versus rostrocaudally, as compared with other scratchactivated interneurons (Holmes and Berkowitz, 2014). T neurons physiologically had briefer action potentials with briefer after- hyperpolarizations, as well as larger scratching membrane potential oscillations, than other scratch-activated interneurons (Berkowitz et al., 2006). All T neurons previously tested were also activated during swimming, making them a subset of multifunctional interneurons (Berkowitz, 2008).

Thus, we examined the current dataset to assess the neurotransmitter(s) used by $\mathrm{T}$ neurons. We defined $\mathrm{T}$ neurons here using the criteria that (1) rostrocaudal dendritic extent/(mediolateral + dorsoventral dendritic extent $)<0.4$ and (2) rostrocaudal soma length/mediolateral soma length $<1.0$, as in the original study (Berkowitz et al., 2006). Cells meeting both criteria were termed $\mathrm{T}(1)$ neurons and cells meeting only the first criterion were termed T(2) neurons (see Materials and Methods). Eight of the 13 multifunctional interneurons with identified neurotransmitter were $\mathrm{T}(1)$ neurons, including four GABAergic or glycinergic interneurons and four glutamatergic interneurons. Four of the 13 were $\mathrm{T}(2)$ neurons, including three that were GABAergic or glycinergic and one that was glutamatergic. Thus, T neurons appear to be heterogeneous with respect to neurotransmitter. The remaining morphologically defined "non- $T$ " multifunctional interneuron was GABAergic or glycinergic.

\section{Motoneuron contacts of multifunctional interneurons}

We found that axon terminals of multifunctional interneurons, in at least some cases, directly contact motoneuron somata and/or dendrites. For example, the multifunctional interneuron shown in Figure 5 was rhythmically activated during rostral scratching and swimming; it was also activated during flexion reflex $(5 A)$. Its soma was in the dorsolateral part of the ventral horn at the D9/D10 border, with axon terminals in the ipsilateral ventral horn (Figs. $5 B, 3 E$ ). It had axon terminals ( $5 C$, red) closely apposed to motoneuron dendrites (ChAT-labeled, blue). These boutons were also closely apposed to gephyrin (white) and not Homer (green) punctate labeling, indicating that this interneuron was GABAergic or glycinergic. The glutamatergic multifunctional interneuron shown in Figure 4, $D$ and $E$, also had boutons (red) closely apposed to a motoneuron dendrite (ChAT, blue). We found close appositions between interneuron axon-terminal boutons and motoneuron somata/dendrites for six of the $13 \mathrm{mul}$ - 
A

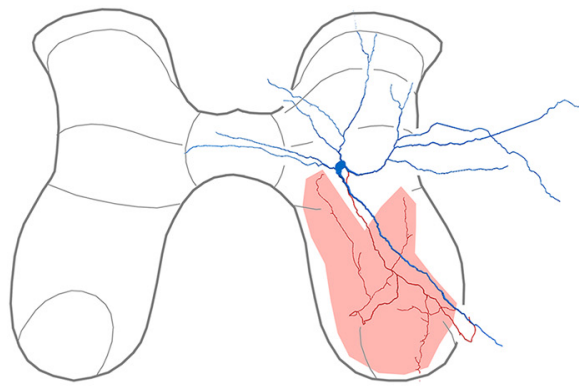

D
B

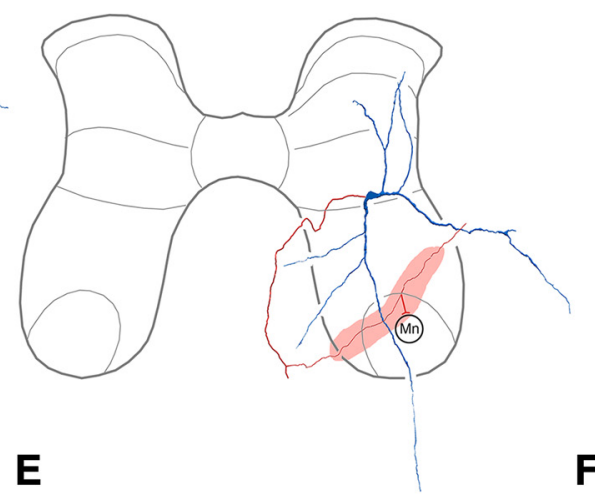

C

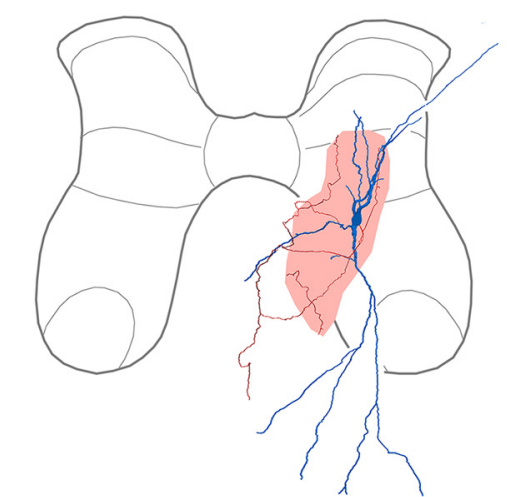

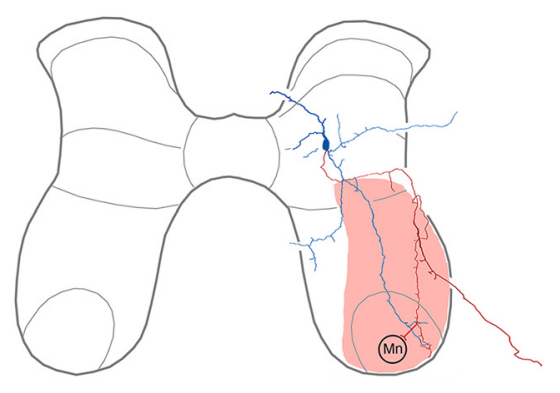

G

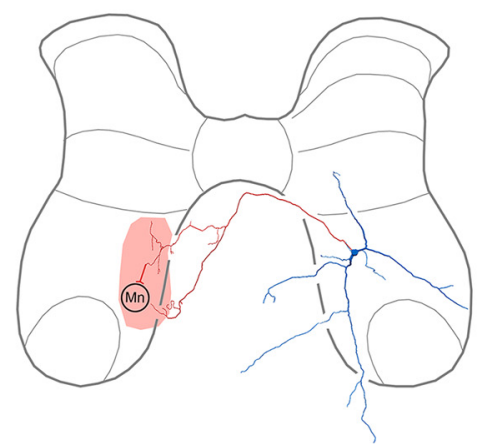

H

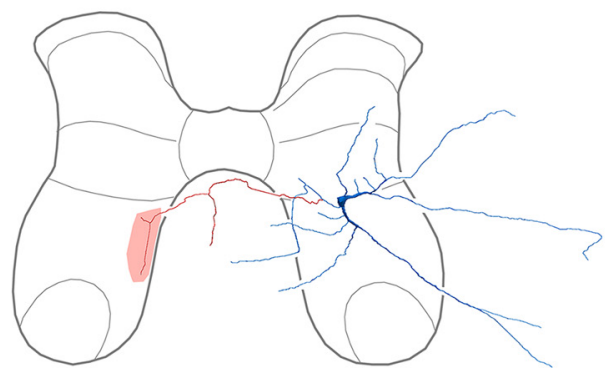

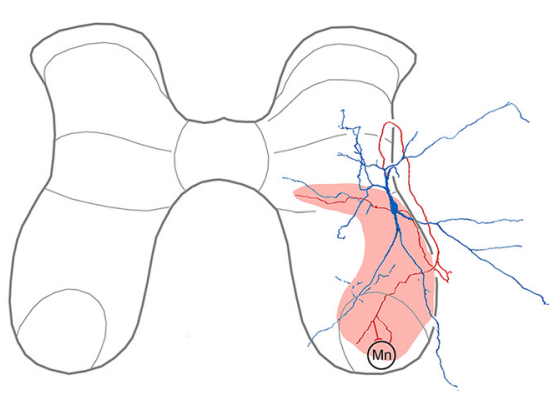
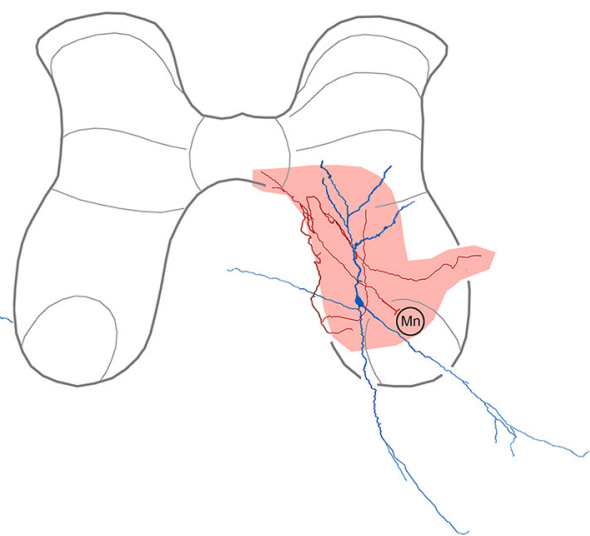

Figure 3. Reconstructions of inhibitory multifunctional interneurons. $\boldsymbol{A}-\boldsymbol{F}$, Interneurons with ipsilateral axon terminals. $\mathbf{G}-\boldsymbol{H}$, Interneurons with contralateral axon terminals.

tifunctional interneurons with reacted axon terminals; 5 of the 6 were GABAergic or glycinergic ( 4 ipsilateral and 1 contralateral axon) and 1 was glutamatergic (contralateral axon).

\section{Neurotransmitters of scratch-specialized interneurons}

We were able to determine the neurotransmitter for four scratchspecialized interneurons (Fig. 6), of which three were excitatory (Fig. 6A-C). Two examples are shown in Figure 7. The interneuron shown in Figure $7 A-C$ was strongly and rhythmically activated during caudal scratching $(7 A$, left). This neuron was hyperpolarized and had its spontaneous activity almost completely stopped during swimming, resuming its firing immediately after swimming (Fig. $7 A$, center). It was also weakly activated during flexion reflex (Fig. $7 A$, right). Its soma was in the dorsomedial D8 ventral horn and it had axon terminals in the contralateral ventral horn (Figs. $7 B, 6 B$ ). Its axon terminals (red) were closely apposed to Homer antibody (green), indicating that it was glutamatergic $(7 C)$. The scratch-specialized interneuron shown in Figure 7, D and $E$, had its soma in the D10 ventrolateral dorsal horn and axon terminals in the ipsilateral ventral horn, as well as a contralateral axon branch with no apparent terminals (7D). This interneuron's terminals (red) were closely apposed to punctate Homer antibody labeling (green), demonstrating that this interneuron was glutamatergic (Fig. $7 E$ ). Collaterals from the fourth scratch specialized interneuron were located in the ipsilateral ventral horn forming terminals closely apposed to gephyrin puncta (Fig. 8C). Two of these four scratch-specialized interneurons were $\mathrm{T}(2)$ neurons (see Materials and Methods, Neuronal reconstruction), despite the fact that $\mathrm{T}$ neurons previously were all found to be multifunctional (Berkowitz, 2008); the other two were non-T neurons. Three of these interneurons had ipsilateral axon terminals; one excitatory interneuron projected contralaterally. Three had peak depolarization during HF bursts (phases of 0.00 , 0.01 and 0.16 ), whereas one was between HF bursts (phase of 0.68).

\section{Motoneuron contacts of scratch-specialized interneurons}

We found that axon terminals of scratch-specialized interneurons also can directly contact motoneuron somata and/or den- 
drites. The interneuron shown in Figure 8 was strongly and rhythmically activated during rostral scratching but was inhibited during swimming and flexion reflex $(8 A)$. Its soma was in the dorsolateral part of the D9 ventral horn, with axon terminals in the ipsilateral ventral horn (Figs. $8 B, 6 D$ ). A number of terminals (Fig. $8 C$, red) were closely apposed to motoneuron dendrites (ChAT-labeled, blue). Close appositions to motoneurons were not found for the other scratch-specialized interneurons.

Axon terminals were also successfully reacted to determine the neurotransmitter for two scratch-activated interneurons that were not tested during swimming (because swimming was not successfully evoked in those animals). One of these interneurons was also activated during flexion reflex; it had an ipsilateral axon and was glutamatergic. The other was not tested during flexion reflex; it had a contralateral axon and was GABAergic or glycinergic. Both were $\mathrm{T}(1)$ neurons. We found close appositions between axonterminals of the former interneuron and motoneuron somata/dendrites.

Projection patterns of multifunctional versus scratch-specialized interneurons The data show that many multifunctional interneurons project to motor nuclei. Contralaterally projecting excitatory and ipsilaterally projecting inhibitory multifunctional interneurons both form extensive terminations within motor nuclei along with more dorsal areas of the ventral horn and the intermediate gray matter. These cells therefore are likely to be lastorder premotor interneurons that contact motoneurons directly but their extensive axonal ramifications within dorsal regions of the ventral horn and the intermediate zone (a known location for many types of premotor interneuron; ZiskindConhaim and Hochman, 2017; Côté et al., 2018) indicate it is likely that they also form connections with other interneurons. The single example of an excitatory multifunctional ipsilaterally projecting interneuron had restricted projections within the intermediate gray matter. Both contralaterally projecting inhibitory multifunctional cells had projections to the medial region of the ventral horn and one of these cells was shown to form synaptic contacts with a motoneuron (Fig. $3 H$ ). It is thus probable that these interneurons contact other interneurons predominantly. The sample of scratch-specialized cells we have is small and it is not possible to form a general overview of them. The
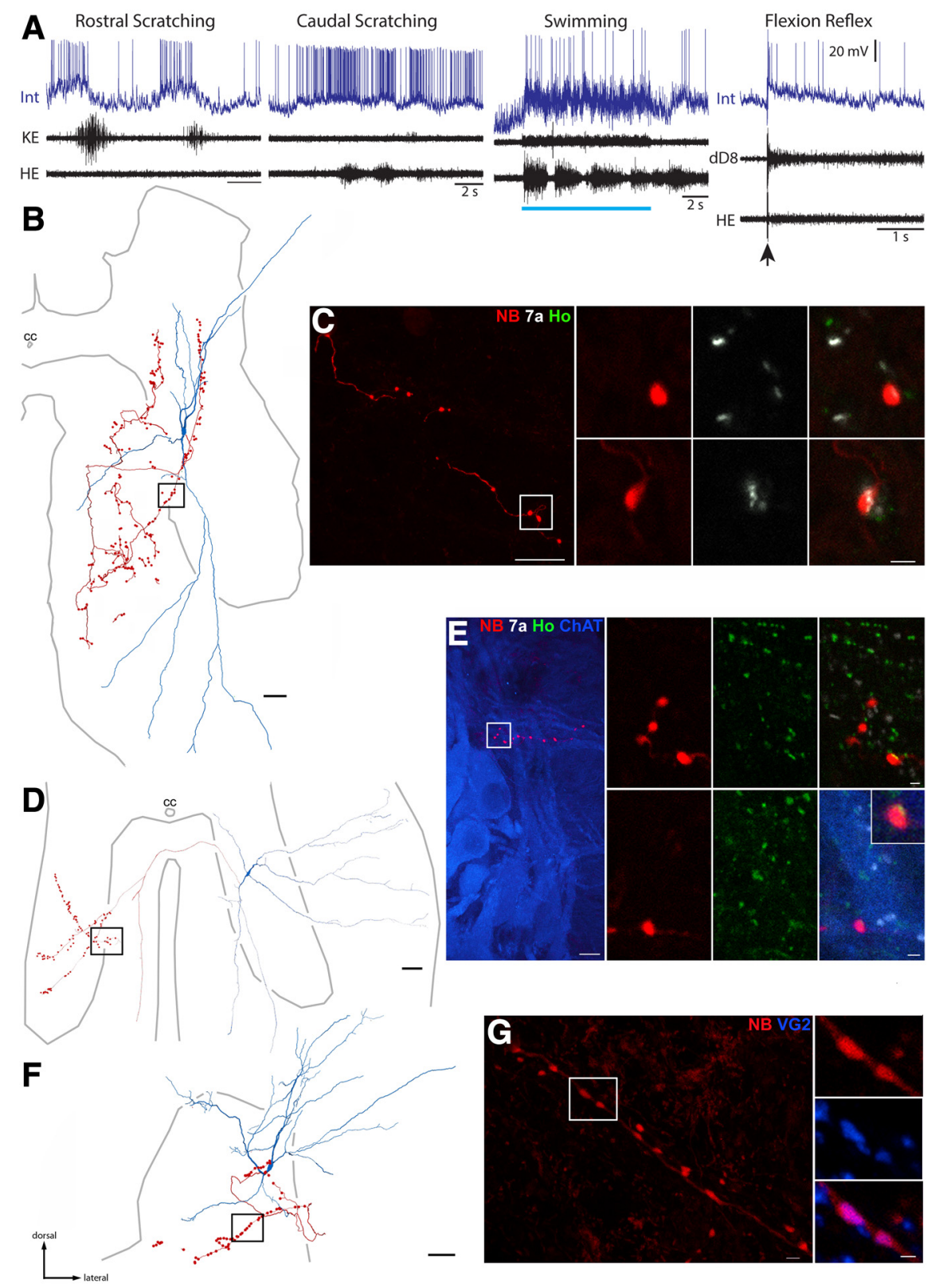

Figure 4. Neurotransmitter identification of multifunctional interneurons. $\boldsymbol{A}-\boldsymbol{C}$, Example of a multifunctional interneuron that was GABAergic or glycinergic. $A$, Intracellular recording from the interneuron (Int) during rostral scratching, caudal scratching, swimming, and flexion reflex motor patterns, monitored via a knee-extensor motor nerve (KE), the HE motor nerve, and the distal D8 (dD8) segmental nerve, the latter of which includes axons of motoneurons activated during HF bursts, as well as others activated during HE bursts (Mortin and Stein, 1989). Blue bar indicates the period of swim-evoking stimulation; arrow indicates the moment of flexion reflex-evoking stimulation. $\boldsymbol{B}$, Reconstruction of the interneuron in $\boldsymbol{A}$, showing its soma and dendrites (blue), along with its axon and axon terminals (red); box indicates the region shown in $\boldsymbol{C}$, left. $\boldsymbol{C}$, Left, Projected image showing NB-labeled (red) axon collateral of the interneuron shown in $\boldsymbol{A}$ and $\boldsymbol{B}$ in the ventral funiculus; box indicates two terminals seen at higher-magnification at right. $C$, Right, Single optical sections $(0.3 \mu \mathrm{m}$ depth) showing NB-labeled terminals (red) and immunoreactions associated with gephyrin (white) and Homer (green), postsynaptic proteins associated with GABAergic/glycinergic and glutamatergic synapses, respectively. Appositions of gephyrin and terminals indicate that this interneuron is inhibitory. Scale bars: left, $10 \mu \mathrm{m}$; right, $1 \mu \mathrm{m}$. $\boldsymbol{D}$, Reconstruction of another multifunctional interneuron, showing its soma and dendrites (blue), along with its axon and axon terminals (red). $\boldsymbol{E}$, Left, Projected image showing a branch of an axon collateral approaching a cluster of motoneurons (labeled with antibodies raised against (hAT; blue) in the contralateral ventral horn. $\boldsymbol{E}$, Right, projected image showing NB and Homer immunoreactivity; two sets of panels (single optical sections) show two terminals associated with Homer (Ho; green), confirming this interneuron is excitatory; bottom right, inset shows one terminal at higher-magnification; bottom right also shows contacts between the interneuron axon (red) and a motoneuron dendrite (blue). Scale bars: left, $10 \mu \mathrm{m}$; right, $1 \mu \mathrm{m}$. $\boldsymbol{F}$, Reconstruction of a third excitatory multifunctional interneuron located in the dorsal horn. $\boldsymbol{G}$, Left, Projected image of a length of axon collateral; right (single optical sections), two terminals were immunoreactive for VGLUT2. cc, Central canal. Image orientation in subsequent figures is the same as shown here. 


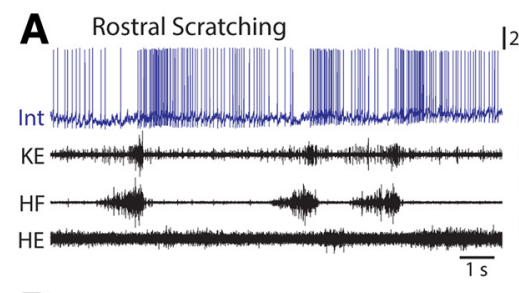

B

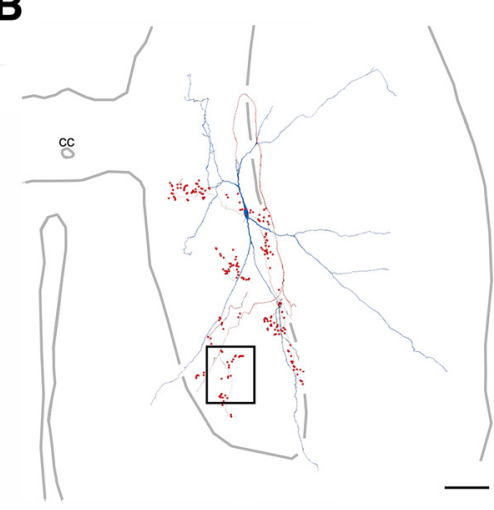

$20 \mathrm{mV} \quad$ Swimming

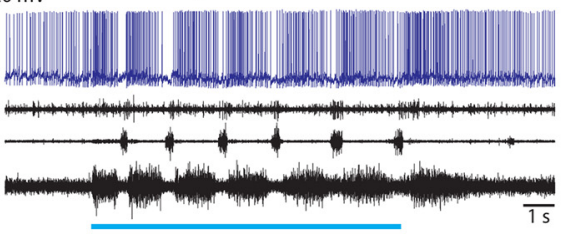

C

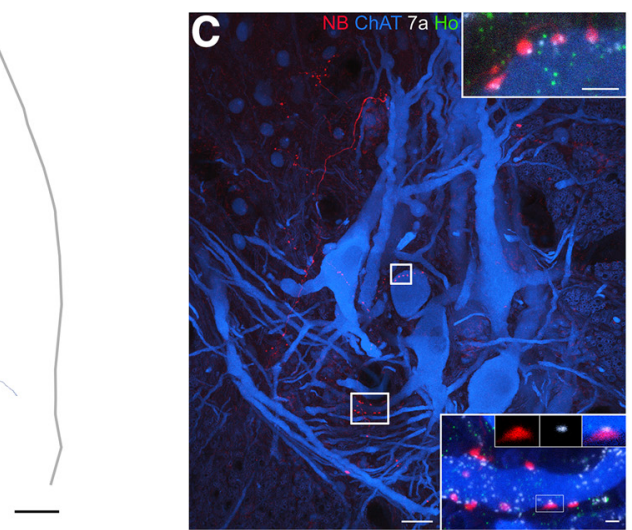

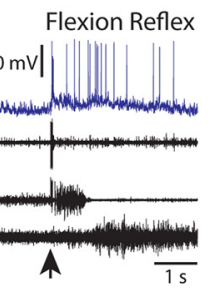

Figure 5. Examples of putative synaptic contacts between a multifunctional interneuron's axon-terminal boutons and motoneuron somata/dendrites. $\boldsymbol{A}$, Intracellular recording from a multifunctional interneuron during rostral scratching, swimming, and flexion reflex motor patterns. Blue bar indicates the period of swim-evoking stimulation; arrow indicates the moment of flexion reflex-evoking stimulation. Int, Interneuron; KE, knee-extensor nerve. $\boldsymbol{B}$, Reconstruction of this interneuron, showing its soma and dendrites (blue), along with its axon and axon terminals (red). $\boldsymbol{C}$, Projected image of a cluster of motoneurons (labeled with antibodies raised against ChAT; blue) and the course followed by an axon collateral (labeled with NB; red) of this interneuron. Boxes indicate areas shown at higher magnification on the right. $\boldsymbol{C}$, Insets, Single optical sections showing contacts between the interneuron axon (red) and motoneuron (blue) soma (top) and dendrites (bottom) with gephyrin (7a; white, boxes) interposed, indicating inhibitory connections. Scale bars: left, $20 \mu \mathrm{m}$; top, $2 \mu \mathrm{m}$; bottom, $1 \mu \mathrm{m}$.

A

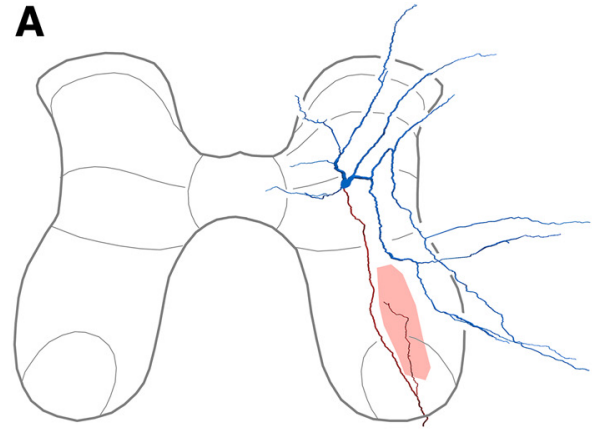

C

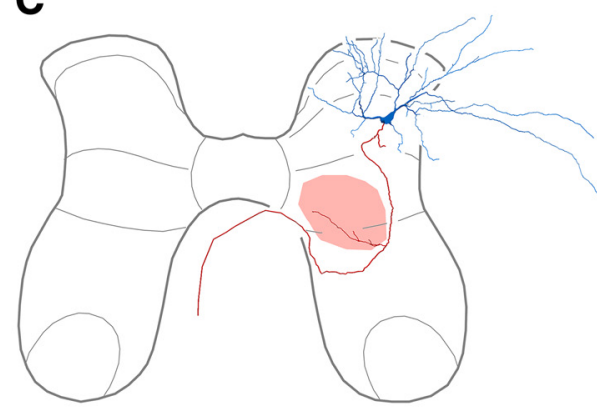

B

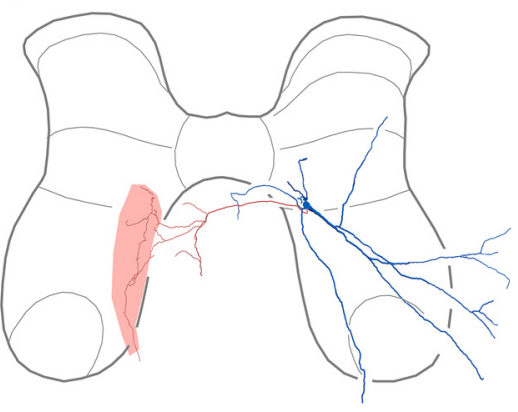

D

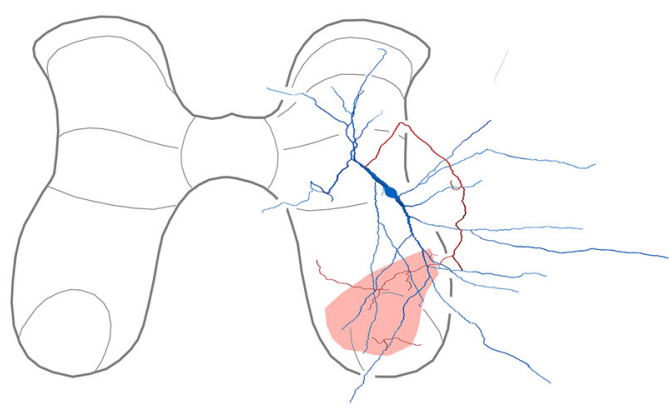

Figure 6. Reconstructions of scratch-specialized interneurons. $A-C$, Excitatory interneurons. D, Inhibitory interneuron.

three excitatory interneurons have projections that tend not to overlap with the motor nucleus whereas the inhibitory cell forms profuse projections within the motor nucleus.

\section{Corollary discharge interneuron}

One additional scratch-specialized interneuron is of special interest (Fig. 9). This interneuron was weakly but rhythmically ac- tivated during pocket scratching, depolarizing late in each HF burst (Fig. 9A). Its soma was in the dorsolateral D9 ventral horn (Fig. 9B). During swimming (Fig. 9C,D), this interneuron fired precisely one action potential following each swim-stimulation pulse to the contralateral D3 lateral funiculus ( $\sim 4 \mathrm{~cm}$ away), at a constant latency of $3.7 \mathrm{~ms}$, with no latency jitter and no depolarization before the action potential (Fig. 9D). This interneuron 
A Caudal Scratching

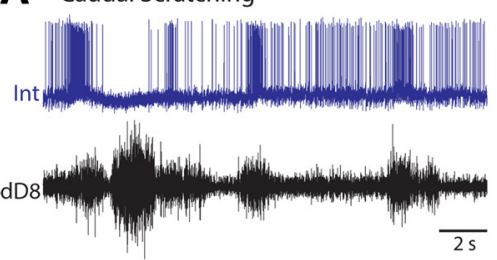

Swimming

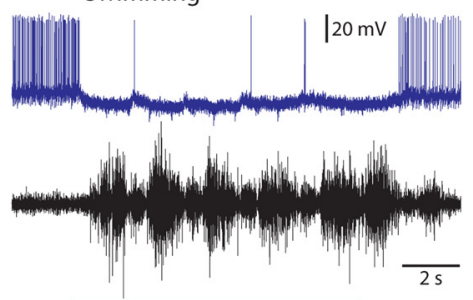

Flexion Reflex

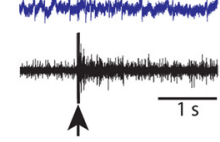

B
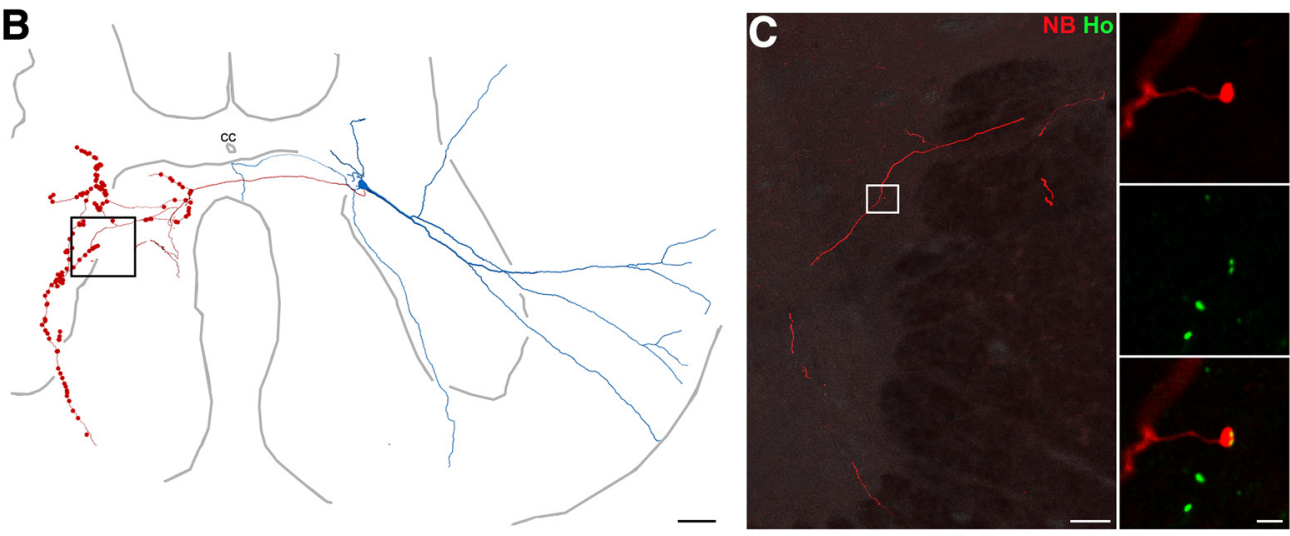

D
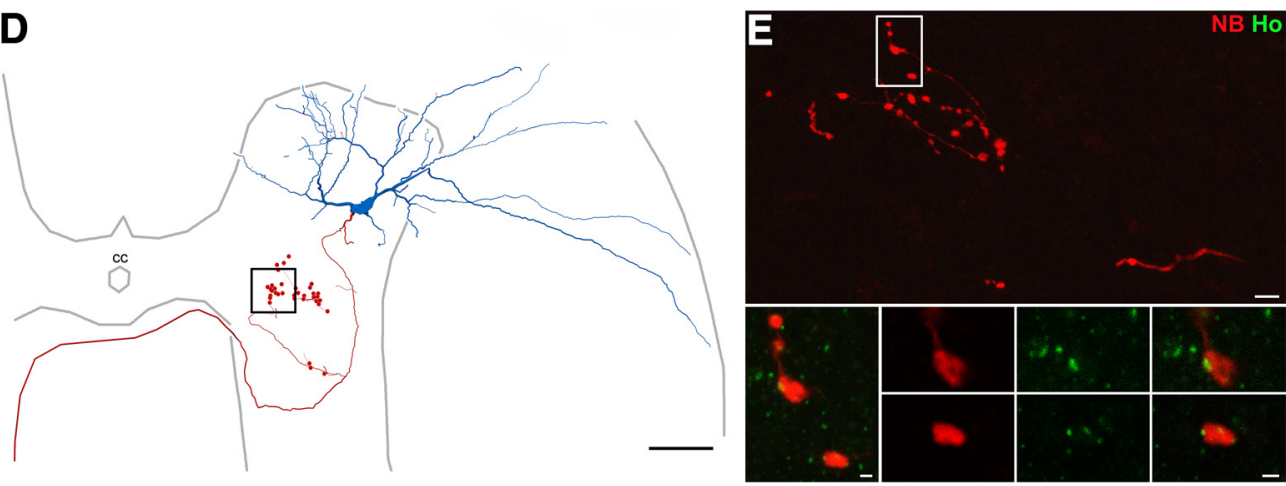

Figure 7. Neurotransmitter identification of scratch-specialized interneurons. $A$, Intracellular recording from an interneuron (Int) during caudal scratching, swimming, and flexion reflex motor patterns, monitored via the dD8 nerve. Blue bar indicates the period of swim-evoking stimulation; arrow indicates the moment of flexion reflex-evoking stimulation. $\boldsymbol{B}$, Reconstruction of this interneuron, showing its soma and dendrites (blue), along with its axon and axon terminals (red); box indicates the region shown in C. C, Course followed by interneuron axon collateral (red); inset, association with Homer (green). Scale bar, $10 \mu \mathrm{m}$. D, Reconstruction of another scratch-specialized interneuron, showing its soma and dendrites (blue), along with its axon and axon terminals (red); box indicates the region shown in $\boldsymbol{E}$, top. $\boldsymbol{E}$, Top, Projected image showing branches of an axon collateral in the medial gray matter just ventral to the central canal; box indicates the region of terminals shown below. $\boldsymbol{E}$, Bottom, Projected image showing NB and Homer immunoreactivity; two sets of panels (single optical sections) show two terminals associated with Homer (Ho; green), confirming this interneuron is excitatory. Scale bars: top, $10 \mu \mathrm{m}$; bottom, $1 \mu \mathrm{m}$.

was not otherwise activated during swimming. This strongly indicates that its action potentials during swim stimulation were antidromically activated via its contralateral ascending axon (with a conduction velocity of $\sim 10 \mathrm{~m} / \mathrm{s}$ ), which must have continued rostrally beyond the D3 segment. Thus, this neuron was not activated during the swimming motor pattern itself, but was rhythmically activated during scratching and was sending its outputs toward the brain. This is consistent with this interneuron providing a corollary discharge signal to the brain during scratching.

\section{Behaviorally specialized motoneurons}

Motoneurons were inadvertently characterized and filled with dyes in this study but their physiological and morphological data in general were not further analyzed. However, there were two motoneurons of special interest that we report on here. Motoneurons in the hindlimb enlargement have all been assumed to be activated during both scratching and swimming (i.e., be multifunctional), as each of these rhythmic behaviors involves alternation between hip flexors and hip extensors, and between knee flexors and knee extensors (Stein, 2005). Flexion reflex also involves hip-flexor activity, along with weak hip-extensor activity at a slight delay (Stein et al., 1982; Currie and Lee, 1996). Thus, it was not expected that any hindlimb-enlargement motoneurons would be behaviorally specialized.

Surprisingly, we found two examples of behaviorally specialized motoneurons (Figs. 10, 11). Figure 10 shows a scratchspecialized motoneuron. Its soma was in the ventrolateral part of the ventral horn (at the border of the D8 and D9 segments), where limb motoneuron somata typically are located (Fig. 10A). It depolarized rhythmically and fired on some cycles during caudal scratching (Fig. 10B, top). During swimming, however, it depolarized rhythmically but did not fire action potentials (Fig. $10 B$, bottom). These responses thus meet the criteria for a 

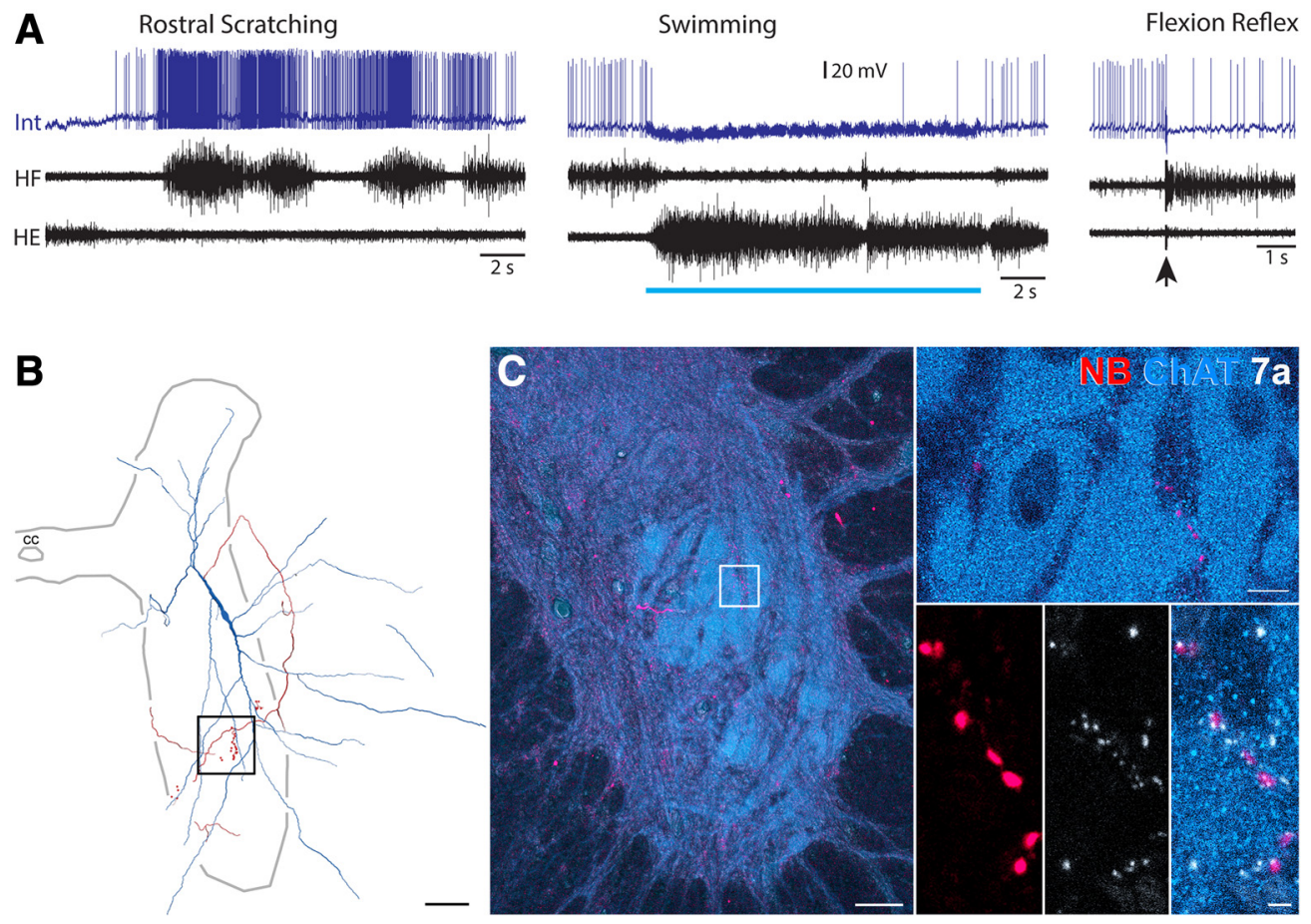

Figure 8. Examples of putative synaptic contacts between a scratch-specialized interneuron's axon-terminal boutons and motoneuron somata. $A$, Intracellular recording from this interneuron during rostral scratching, swimming, and flexion reflex motor patterns. Blue bar indicates the period of swim-evoking stimulation; arrow indicates the moment of flexion reflex-evoking stimulation. Int, Interneuron. B, Reconstruction of this interneuron, showing its soma and dendrites (blue), along with its axon and axon terminals (red); box indicates the region shown in $\boldsymbol{C}$, left. $\boldsymbol{C}$, Left, Low-power projected images of a motor nucleus in the ventral horn (labeled with antibodies raised against ChAT; blue) and a collateral from the interneuron (red). $\boldsymbol{C}$, Top right, Single optical section showing arrangement of motoneurons. C, Bottom right, Series of single optical sections at intervals through the motor nucleus showing putative contacts between the interneuron collateral and motoneurons with gephyrin (white) interposed between them. Scale bars: left, $50 \mu \mathrm{m}$; top right, $10 \mu \mathrm{m}$; bottom right, $2 \mu \mathrm{m}$.

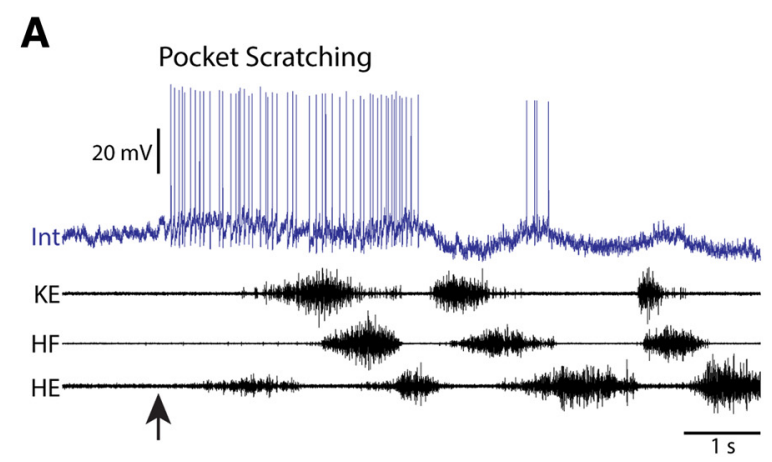

\section{C}

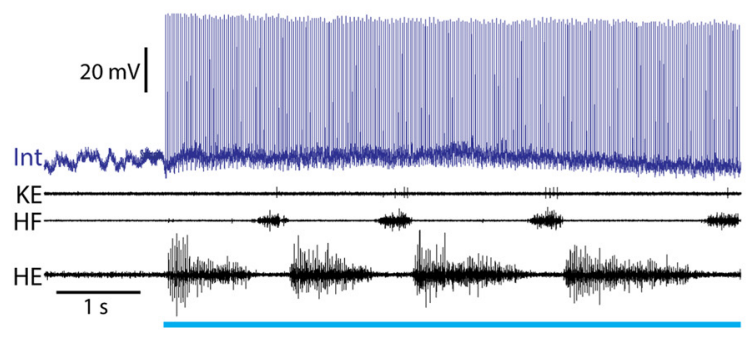

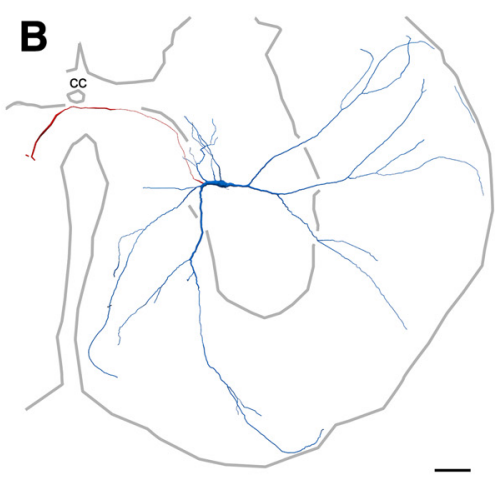

D Swim Stimulation

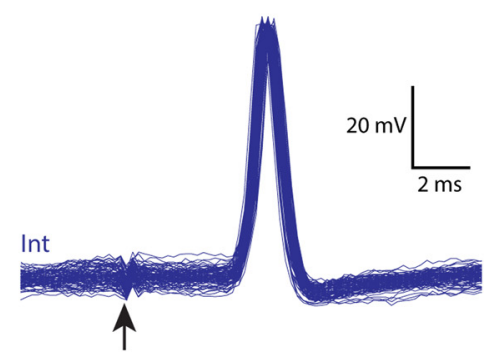

Figure 9. A scratch-specialized interneuron with an axon ascending in the contralateral D3 lateral funiculus. A, Intracellular recording from this interneuron during pocket scratching; arrow indicates stimulus onset. $\boldsymbol{B}$, Reconstruction of this interneuron, showing its soma and dendrites (blue), along with its axon and axon terminals (red). $\boldsymbol{C}$, Intracellular recording from this interneuron during swimming; blue bar indicates the period of swim-evoking stimulation of the contralateral D3 lateral funiculus. $\boldsymbol{D}$, Superimposed traces of this interneuron's response to the first 100 swim-evoking stimulation pulses at $40 \mathrm{~Hz}$. Arrow indicates moment of stimulation. Note that the interneuron responds at the same latency to each pulse with no jitter and no depolarization before the action potential onset, indicating that it was antidromically activated. Int, Interneuron; KE, knee-extensor nerve. 
scratch-specialized neuron (Berkowitz, 2002). This motoneuron's action potentials occurred late within HF nerve bursts. It may have innervated a muscle that is more strongly activated during scratching than swimming, such as a hip flexor. Note, however, that the HF nerve burst in these examples is just as large in mean amplitude (though not as long) during swim$\operatorname{ming}(6.81 \pm 0.75 \mu \mathrm{V})$ as during caudal scratching $(5.68 \pm 1.05 \mu \mathrm{V} ; p=0.11$, Mann-Whitney test), yet this motoneuron only reaches action potential threshold during scratching.

Figure 11 shows a flexion reflexselective motoneuron. Its soma was in the D10 segment, in the middle of the hindlimb enlargement rostrocaudally, but on the medial edge of the ventral horn (Fig. 11A), a location more medial than expected for limb motoneurons, though within the range of labeled motoneuron soma locations previously seen following application of a retrograde tracer to the D8 ventral root (Berkowitz and Stein, 1994a). This motoneuron generated no action potentials during scratching (Fig. $11 B$ ) or swimming (11C) and appeared to receive no rhythmic drive during either of these rhythmic motor patterns. Nonetheless, this motoneuron was very strongly activated during flexion reflex (Fig. 11D), beginning within $\sim 30 \mathrm{~ms}$ of the first electrical foot stimulus $(11 E)$, well before the HF nerve burst began, and continuing for several seconds, along with the flexion reflex motor pattern in the HF nerve. There was no sign of this motoneuron's action potential within a spike-triggered average of the HF nerve recording (Fig. 11F).

\section{Discussion}

We report here for the first time the neurotransmitters and putative synaptic contacts with motoneurons of multifunctional (scratch/swim) and scratchspecialized turtle spinal interneurons. We also report for the first time the unexpected existence of behaviorally specialized spinal motoneurons in the turtle hindlimb enlargement.

\section{Multifunctional spinal interneurons}

We found that multifunctional interneurons were heterogeneous with respect to neurotransmitter. Some were glutamatergic and thus likely excitatory, whereas others were GABAergic or glycinergic and thus likely inhibitory. The multifunctional interneurons that met the morphological criteria to be transverse interneurons (T neurons; Berkowitz et al., 2006) also included both excitatory and inhibitory interneurons. Morphologically defined T(1) neurons made up a larger fraction $(62 \%)$ of the scratch-activated interneurons in this study than in the original study (36\%; Berkowitz et al., 2006), perhaps because ron; KE, knee-extensor nerve.
A

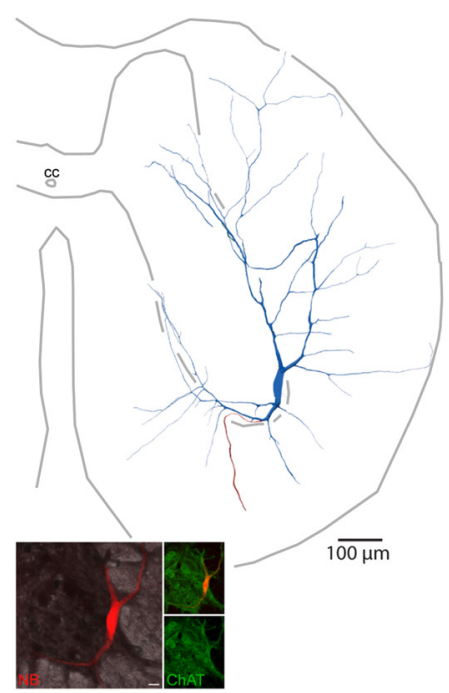

B Caudal Scratching
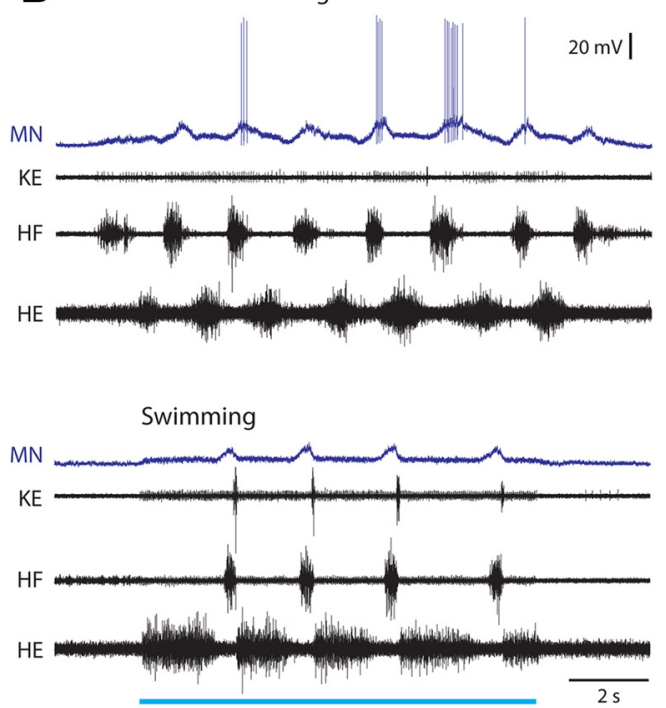

Figure 10. Scratch-specialized motoneuron. A, Reconstruction of this motoneuron, showing its soma and dendrites (blue), along with its axon (red). Inset, ChAT-immunoreactivity (green) associated with the soma (red). $\boldsymbol{B}$, Intracellular recording from this motoneuron during caudal scratching and swimming. Blue bar indicates the period of swim-evoking stimulation. MN, Motoneu-
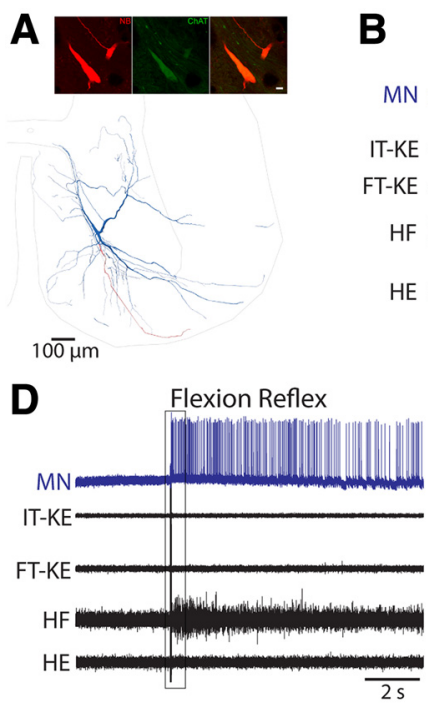

$\mathbf{F}$

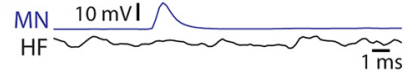

B
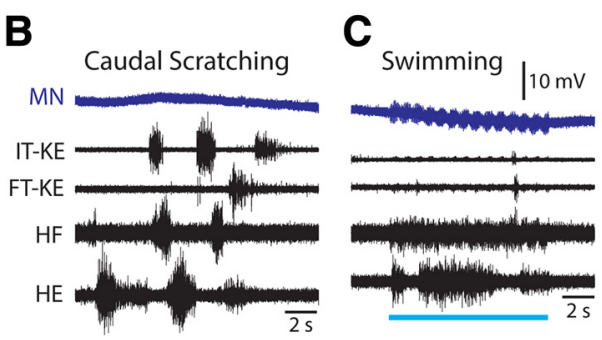

E

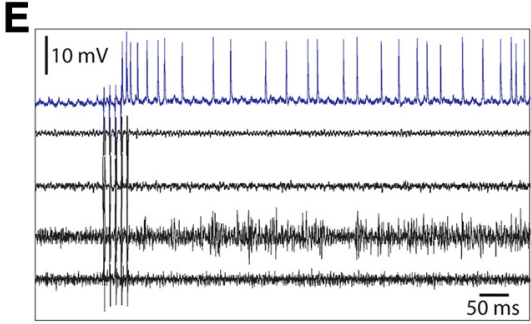

Figure 11. Flexion reflex-selective motoneuron. $A$, Reconstruction of this motoneuron, showing its soma and dendrites (blue), along with its axon (red); inset, ChAT immunoreactivity (green) associated with soma and dendrites (red). B, C, Intracellular recording from this motoneuron during caudal scratching $(\boldsymbol{B})$ and swimming $(\boldsymbol{C})$. Blue bar indicates the period of swim-evoking stimulation. $\boldsymbol{D}$, Intracellular recording from this motoneuron during flexion reflex; boxed region expanded in $\boldsymbol{E}$. $\boldsymbol{F}$, Spike-triggered average (from 195 intracellular motoneuron spikes) of the $\mathrm{HF}$ nerve activity along with the intracellular spike. MN, Motoneuron; IT-KE and FT-KE, knee-extensor nerves.

in this study, we needed to inject $\sim 10$ times as much current to adequately label axon terminals for immunocytochemistry as we previously injected to label the soma and dendrites (and sometimes terminals; Berkowitz et al., 2006). Injecting much more current may have labeled thin dendritic branches that were not successfully labeled in the earlier study and which substantially increased the total dendritic extent in the transverse plane for what previously would have been classified as non-T interneurons. T-neuron dendrites are thick and rela- 


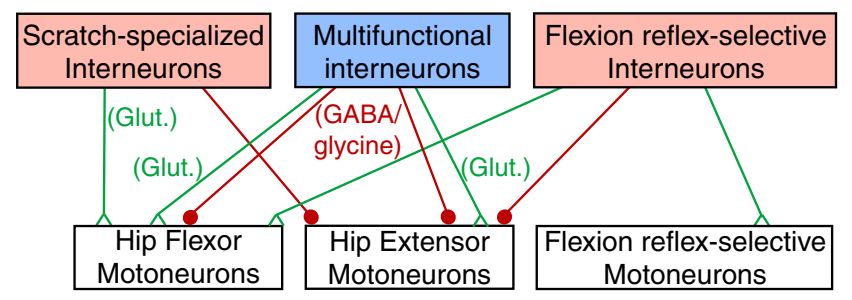

Figure 12. Diagram illustrating possible circuitry consistent with the current data. Green triangles indicate excitatory chemical synapses and red filled circles indicate inhibitory chemical synapses. Glut, Glutamatergic.

tively unbranched (Berkowitz et al., 2006; Holmes and Berkowitz, 2014), so they would be apparent even with less dye. Thus, additional criteria need to be applied to define this morphological-physiological category of interneurons precisely.

Multifunctional interneurons, including T neurons, that are rhythmically activated during swimming and scratching are good candidates to contribute to central pattern generation for multiple types of rhythmic motor patterns. Previous evidence suggested that the spinal CPG networks for swimming and all three forms of scratching are largely shared (Berkowitz and Stein, 1994b; Berkowitz, 2001b, 2002, 2008; Hao et al., 2011; Hao and Berkowitz, 2017). Combined with the current findings, it seems likely that these CPG networks include both excitatory and inhibitory interneurons. Previous evidence suggested that inhibitory interneurons might play a larger role than excitatory interneurons in these CPG networks, because scratch-activated interneurons were more rhythmic when they fired at lower rates (Berkowitz and Stein, 1994b) and because the trough phases of interneuron membrane potential oscillations were significantly correlated between swimming and scratching even though the peak phases were not (Berkowitz, 2008). In the current study, most multifunctional interneurons with identified neurotransmitter were inhibitory, consistent with inhibition playing a larger role than excitation in these $\mathrm{CPG}$ networks. A recent study also encountered predominantly inhibitory local interneurons in recordings from the turtle spinal cord (Radosevic et al., 2019). More generally, a segment of the turtle spinal cord hindlimb enlargement contains $\sim 7000$ interneurons with axons projecting at least one segment, which is $\sim 5$ times as many as there are motoneurons in the segment (Nissen et al., 2008), but the proportion of interneurons that is inhibitory is not known. In the largest segment of the turtle hindlimb enlargement, there are $\sim 16,000$ interneurons in total (Walløe et al., 2011).

For 6 of the 13 multifunctional interneurons with NB-labeled axon terminals, we found close appositions between terminals and motoneurons. This is likely an underrepresentation, because we could only find well labeled axon terminals within $\sim 1 \mathrm{~mm}$ of the interneuron soma. Turtle propriospinal interneuron axons often extend for several millimeters or even centimeters (Berkowitz and Stein, 1994a; Berkowitz, 2004; Nissen et al., 2008). Thus, it is likely that many or most multifunctional interneurons directly contact motoneurons, perhaps in addition to having other postsynaptic targets. Many multifunctional interneurons may both contribute to rhythm generation of locomotion and scratching and be last-order premotor interneurons, with some inhibitory and others excitatory (Fig. 12). That many multifunctional interneurons directly contact motoneurons is consistent with our recent finding that the scratching and swimming CPGs largely converge before motoneurons (Hao and Berkowitz, 2017).
In hatchling tadpoles, both types of multifunctional premotor interneurons activated during swimming and struggling motor patterns, commissural interneurons and ascending interneurons, are glycinergic and inhibitory (Li et al., 2007; Berkowitz et al., 2010). In larval zebrafish, a class of multifunctional premotor interneurons activated during swimming, struggling, and escape motor patterns, commissural bifurcating longitudinal interneurons, is also inhibitory (Liao and Fetcho, 2008). Also in larval zebrafish, however, dorsal circumferential descending (V2a) interneurons are excitatory and premotor; they are activated during most escapes as well as fast swimming, though not during slow swimming (Ritter et al., 2001; Bhatt et al., 2007). In cats, $\sim$ two-thirds of synaptic terminals on spinal motoneurons are inhibitory (Ornung et al., 1996). Both Renshaw cells (McCrea et al., 1980; Deliagina and Feldman, 1981; Pratt and Jordan, 1987) and Ia inhibitory interneurons (Geertsen et al., 2011) are premotor interneurons that are rhythmically activated during both locomotor and scratching motor patterns and are also inhibitory. Thus, across vertebrates, multifunctional premotor spinal interneurons seem to be predominantly inhibitory.

\section{Scratch-specialized spinal interneurons}

Three of the four scratch-specialized interneurons for which we were able to determine the neurotransmitter were glutamatergic; two met the morphological criteria for $\mathrm{T}(2)$ [but not $\mathrm{T}(1)$ ] neurons, though all $\mathrm{T}$ neurons previously tested during both scratching and swimming were multifunctional (Berkowitz, 2008). We found close appositions between interneuron terminals and motoneurons for one scratch-specialized interneuron, ipsilaterally. Three of the scratch-specialized interneurons successfully tested in this study were excitatory and one was inhibitory. Together, these findings suggest that some scratch-specialized interneurons may directly excite motoneurons that are more strongly activated during scratching than swimming, such as ipsilateral hip-flexor motoneurons (or contralateral hip-extensor motoneurons), whereas others may directly inhibit motoneurons that are less strongly activated during scratching than swimming, such as ipsilateral hip-extensor motoneurons (or contralateral hip-flexor motoneurons; Juranek and Currie, 2000; Berkowitz, 2002, 2008; Hao et al., 2011; Hao and Berkowitz, 2017). If ipsilateral hip-flexor motoneurons receive excitation from both multifunctional and scratch-specialized interneurons during scratching, but only from multifunctional interneurons during swimming, this could explain why ipsilateral hip-flexor motoneurons are more strongly activated during scratching than swimming (Fig. 12).

In hatchling tadpoles, the two types of behaviorally specialized (for swimming or struggling) premotor interneurons, descending interneurons and repetitive-firing descending interneurons, respectively, are both glutamatergic (and in the case of descending interneurons, also cholinergic) and excitatory with ipsilateral axons (Berkowitz et al., 2010), similar to most of the scratchspecialized interneurons described here. In addition to these premotor interneurons, tadpoles also have struggling-specialized excitatory commissural interneurons, which are also glutamatergic (Berkowitz et al., 2010). In larval zebrafish, swimmingspecialized commissural primary ascending interneurons are also glutamatergic and excitatory (but not premotor; Berkowitz et al., 2010). However, the behaviorally specialized premotor interneurons, struggling-specialized commissural longitudinal ascending interneurons and escape-specialized commissural local interneurons, are both glycinergic and inhibitory (Liao and Fetcho, 2008; Berkowitz et al., 2010). 
One scratch-specialized interneuron in this study (Fig. 9) was antidromically activated by each (swim-triggering) electrical pulse in the contralateral lateral funiculus, consistent with it sending a scratch-related corollary discharge signal rostrally, perhaps to the brain. Corollary discharge locomotor signals are sent from the spinal cord to the brain in cats via ventral spinocerebellar tract (VSCT) neurons during locomotion (Arshavsky et al., 1972) and scratching (Arshavsky et al., 1978). Cat VSCT axons ascend contralaterally, in the lateral funiculus, like the interneuron described here, and typically are activated during hip-flexor bursts, also like the interneuron described here.

\section{Behaviorally specialized motoneurons}

We found two behaviorally specialized hindlimb-enlargement motoneurons, one scratch-specialized and the other flexion reflex-selective. This was surprising because motor nerves and muscles studied to date are all activated during all three forms of turtle scratching and swimming (Lennard and Stein, 1977; Robertson et al., 1985; Juranek and Currie, 2000; Berkowitz, 2002, 2008; Hao et al., 2011; Hao and Berkowitz, 2017); hip-flexor and, to a lesser extent, hip-extensor motor nerves are also activated during flexion reflex (Stein et al., 1982; Currie and Lee, 1996). The general assumption has been that all hindlimb muscles contribute to all hindlimb behaviors. Typically, the largest motoneurons within a motor pool are only recruited when the motor pool is most strongly activated (Henneman, 1957). Thus, some hipflexor motoneurons may be recruited during scratching but not swimming. Such motoneurons could be excited partly by scratch-specialized interneurons (see previous section; Fig. 12). The scratch-specialized motoneuron described here, however, was activated during scratching but not swimming even though the amplitude of the HF burst was just as large (though its duration was less) during swimming as during scratching.

Another possibility is that there are muscles, innervated by hindlimb-enlargement motoneurons, that are only activated during a subset of hindlimb behaviors. This hypothesis is more consistent with the flexion reflex-selective motoneuron described here, because the HF nerve and muscle are strongly activated during scratching and flexion reflex, so it would be surprising if an HF motoneuron was strongly activated during flexion reflex but did not even receive rhythmic depolarization during scratching; in addition, this motoneuron began firing before the HF nerve and there was no sign of its spike in the HF nerve recording. Our previous finding that there are flexion reflex-selective spinal interneurons (Berkowitz, 2007), including some that are inhibited during the flexor phase of scratching and swimming, and our current finding that there are flexion reflex-specific motoneurons suggest that foot stimulation that triggers flexion reflex may in part act via its own pathway to flexor motoneurons, separate from the swimming and scratching CPGs. Such a "private" flexion reflex pathway would contrast with the long-held idea that flexion reflex afferents generally have their effects via activation of a flexor module of a locomotor and scratching CPG (Jankowska et al., 1967; Lundberg, 1979; Ostry et al., 1991; Sandrini et al., 2005).

\section{References}

Alaburda A, Hounsgaard J (2003) Metabotropic modulation of motoneurons by scratch-like spinal network activity. J Neurosci 23:8625-8629.

Alaburda A, Russo R, MacAulay N, Hounsgaard J (2005) Periodic highconductance states in spinal neurons during scratch-like network activity in adult turtles. J Neurosci 25:6316-6321.

Ambalavanar R, Morris R (1989) Fluoro-gold injected either subcutane- ously or intravascularly results in extensive retrograde labelling of CNS neurones having axons terminating outside the blood-brain barrier. Brain Res 505:171-175.

Arshavsky YI, Berkinblit MB, Fukson OI, Gelfand IM, Orlovsky GN (1972) Origin of modulation in neurones of the ventral spinocerebellar tract during locomotion. Brain Res 43:276-279.

Arshavsky YI, Gelfand IM, Orlovsky GN, Pavlova GA (1978) Messages conveyed by spinocerebellar pathways during scratching in the cat: II. Activity of neurons of the ventral spinocerebellar tract. Brain Res 151:493-506.

Berg RW, Alaburda A, Hounsgaard J (2007) Balanced inhibition and excitation drive spike activity in spinal half-centers. Science 315:390-393.

Berkowitz A (2001a) Broadly tuned spinal neurons for each form of fictive scratching in spinal turtles. J Neurophysiol 86:1017-1025.

Berkowitz A (2001b) Rhythmicity of spinal neurons activated during each form of fictive scratching in spinal turtles. J Neurophysiol 86:1026-1036.

Berkowitz A (2002) Both shared and specialized spinal circuitry for scratching and swimming in turtles. J Comp Physiol A Neuroethol Sens Neural Behav Physiol 188:225-234.

Berkowitz A (2004) Propriospinal projections to the ventral horn of the rostral and caudal hindlimb enlargement in turtles. Brain Res 1014: $164-176$.

Berkowitz A (2005) Physiology and morphology indicate that individual spinal interneurons contribute to diverse limb movements. J Neurophysiol 94:4455-4470.

Berkowitz A (2007) Spinal interneurons that are selectively activated during fictive flexion reflex. J Neurosci 27:4634-4641.

Berkowitz A (2008) Physiology and morphology of shared and specialized spinal interneurons for locomotion and scratching. J Neurophysiol 99: 2887-2901.

Berkowitz A (2010) Multifunctional and specialized spinal interneurons for turtle limb movements. Ann N Y Acad Sci 1198:119-132.

Berkowitz A, Stein PS (1994a) Descending propriospinal axons in the hindlimb enlargement of the red-eared turtle: cells of origin and funicular courses. J Comp Neurol 346:321-336.

Berkowitz A, Stein PS (1994b) Activity of descending propriospinal axons in the turtle hindlimb enlargement during two forms of fictive scratching: phase analyses. J Neurosci 14:5105-5119.

Berkowitz A, Yosten GL, Ballard RM (2006) Somato-dendritic morphology predicts physiology for neurons that contribute to several kinds of limb movements. J Neurophysiol 95:2821-2831.

Berkowitz A, Roberts A, Soffe SR (2010) Roles for multifunctional and specialized spinal interneurons during motor pattern generation in tadpoles, zebrafish larvae, and turtles. Front Behav Neurosci 4:36.

Berkowitz A, Bannatyne BA, Dyer GMC, Maxwell DJ (2018) Neurotransmitters and motoneuron contacts of multifunctional and behaviorally specialized turtle spinal cord interneurons. Paper presented at International Congress of Neuroethology, Brisbane, Australia, July.

Bhatt DH, McLean DL, Hale ME, Fetcho JR (2007) Grading movement strength by changes in firing intensity versus recruitment of spinal interneurons. Neuron 53:91-102.

Briggman KL, Kristan WB (2008) Multifunctional pattern-generating circuits. Annu Rev Neurosci 31:271-294.

Côté MP, Murray LM, Knikou M (2018) Spinal control of locomotion: individual neurons, their circuits and functions. Front Physiol 9:784.

Currie SN, Gonsalves GG (1997) Right-left interactions between rostral scratch networks generate rhythmicity in the preenlargement spinal cord of the turtle. J Neurophysiol 78:3479-3483.

Currie SN, Lee S (1996) Glycinergic inhibition in the turtle spinal cord regulates the intensity and pattern of fictive flexion reflex motor output. Neurosci Lett 205:75-78.

Currie SN, Stein PS (1989) Interruptions of fictive scratch motor rhythms by activation of cutaneous flexion reflex afferents in the turtle. J Neurosci 9:488-496.

Currie SN, Stein PS (1990) Cutaneous stimulation evokes long-lasting excitation of spinal interneurons in the turtle. J Neurophysiol 64:1134-1148.

Delgado-Lezama R, Perrier JF, Nedergaard S, Svirskis G, Hounsgaard J (1997) Metabotropic synaptic regulation of intrinsic response properties of turtle spinal motoneurones. J Physiol 504:97-102.

Delgado-Lezama R, Perrier JF, Hounsgaard J (1999) Local facilitation of 
plateau potentials in dendrites of turtle motoneurones by synaptic activation of metabotropic receptors. J Physiol 515:203-207.

Deliagina TG, Feldman AG (1981) Activity of Renshaw cells during fictive scratch reflex in cat. Exp Brain Res 42:108-115.

Earhart GM, Stein PS (2000) Scratch-swim hybrids in the spinal turtle: blending of rostral scratch and forward swim. J Neurophysiol 83:156165.

Elson MS, Berkowitz A (2016) Flexion reflex can interrupt and reset the swimming rhythm. J Neurosci 36:2819-2826.

Essrich C, Lorez M, Benson JA, Fritschy JM, Lüscher B (1998) Postsynaptic clustering of major GABAA receptor subtypes requires the gamma 2 subunit and gephyrin. Nat Neurosci 1:563-571.

Geertsen SS, Stecina K, Meehan CF, Nielsen JB, Hultborn H (2011) Reciprocal Ia inhibition contributes to motoneuronal hyperpolarisation during the inactive phase of locomotion and scratching in the cat. J Physiol 589:119-134.

Guertin PA, Hounsgaard J (1998) NMDA-induced intrinsic voltage oscillations depend on L-type calcium channels in spinal motoneurons of adult turtles. J Neurophysiol 80:3380-3382.

Gutierrez-Mecinas M, Kuehn ED, Abraira VE, Polgár E, Watanabe M, Todd AJ (2016) Immunostaining for Homer reveals the majority of excitatory synapses in laminae I-III of the mouse spinal dorsal horn. Neuroscience 329:171-181.

Guzulaitis R, Hounsgaard J (2017) Synaptic excitation in spinal motoneurons alternates with synaptic inhibition and is balanced by outward rectification during rhythmic motor network activity. J Neurosci 37: 9239-9248.

Guzulaitis R, Alaburda A, Hounsgaard J (2013) Increased activity of premotor network does not change the excitability of motoneurons during protracted scratch initiation. J Physiol 591:1851-1858.

Guzulaitis R, Alaburda A, Hounsgaard J (2014) Dense distributed processing in a hindlimb scratch motor network. J Neurosci 34:10756-10764.

Hao ZZ, Berkowitz A (2017) Shared components of rhythm generation for locomotion and scratching exist prior to motoneurons. Front Neural Circuits 11:54.

Hao ZZ, Spardy LE, Nguyen EB, Rubin JE, Berkowitz A (2011) Strong interactions between spinal cord networks for locomotion and scratching. J Neurophysiol 106:1766-1781.

Hao ZZ, Meier ML, Berkowitz A (2014) Rostral spinal cord segments are sufficient to generate a rhythm for both locomotion and scratching but affect their hip extensor phases differently. J Neurophysiol 112:147-155.

Henneman E (1957) Relation between size of neurons and their susceptibility to discharge. Science 126:1345-1347.

Holmes JR, Berkowitz A (2014) Dendritic orientation and branching distinguish a class of multifunctional turtle spinal interneurons. Front Neural Circuits 8:136.

Hounsgaard J, Kiehn O (1989) Serotonin-induced bistability of turtle motoneurones caused by a nifedipine-sensitive calcium plateau potential. J Physiol 414:265-282.

Hounsgaard J, Kjaerulff O (1992) $\mathrm{Ca}^{2+}$-mediated plateau potentials in a subpopulation of interneurons in the ventral horn of the turtle spinal cord. Eur J Neurosci 4:183-188.

Hounsgaard J, Nicholson C (1990) The isolated turtle brain and the physiology of neuronal circuits. In: Preparations of vertebrate central nervous system in vitro (Jahnsen H, ed), pp 155-181. New York: Wiley.

Jankowska E, Jukes MG, Lund S, Lundberg A (1967) The effect of DOPA on the spinal cord: 5. Reciprocal organization of pathways transmitting excitatory action to alpha motoneurones of flexors and extensors. Acta Physiol Scand 70:369-388.

Johnson KP, Tran SM, Siegrist EA, Paidimarri KB, Elson MS, Berkowitz A (2017) Turtle flexion reflex motor patterns show windup, mediated partly by L-type calcium channels. Front Neural Circuits 11:83.

Juranek J, Currie SN (2000) Electrically evoked fictive swimming in the lowspinal immobilized turtle. J Neurophysiol 83:146-155.

Lee EJ, Merwine DK, Padilla M, Grzywacz NM (2007) Choline acetyltransferase-immunoreactive neurons in the retina of normal and darkreared turtle. J Comp Neurol 503:768-778.

Lennard PR, Stein PS (1977) Swimming movements elicited by electrical stimulation of turtle spinal cord: I. Low-spinal and intact preparations. J Neurophysiol 40:768-778.

Liao JC, Fetcho JR (2008) Shared versus specialized glycinergic spinal in- terneurons in axial motor circuits of larval zebrafish. J Neurosci 28: 12982-12992.

Li WC, Sautois B, Roberts A, Soffe SR (2007) Reconfiguration of a vertebrate motor network: specific neuron recruitment and contextdependent synaptic plasticity. J Neurosci 27:12267-12276.

Lundberg A (1979) Multisensory control of spinal reflex pathways. Prog Brain Res 50:11-28.

Lutz PL, Milton SL (2004) Negotiating brain anoxia survival in the turtle. J Exp Biol 207:3141-3147.

McCrea DA, Pratt CA, Jordan LM (1980) Renshaw cell activity and recurrent effects on motoneurons during fictive locomotion. J Neurophysiol 44:475-488.

Mortin LI, Keifer J, Stein PS (1985) Three forms of the scratch reflex in the spinal turtle: movement analyses. J Neurophysiol 53:1501-1516.

Mortin LI, Stein PS (1989) Spinal cord segments containing key elements of the central pattern generators for three forms of scratch reflex in the turtle. J Neurosci 9:2285-2296.

Morton DW, Chiel HJ (1994) Neural architectures for adaptive behavior. Trends Neurosci 17:413-420.

Nissen UV, Moldovan M, Hounsgaard J, Glover JC (2008) Organization of projection-specific interneurons in the spinal cord of the red-eared turtle. Brain Behav Evol 72:179-191.

Ornung G, Shupliakov O, Lindå H, Ottersen OP, Storm-Mathisen J, Ulfhake B, Cullheim S (1996) Qualitative and quantitative analysis of glycineand GABA-immunoreactive nerve terminals on motoneuron cell bodies in the cat spinal cord: a postembedding electron microscopic study. J Comp Neurol 365:413-426.

Ostry DJ, Feldman AG, Flanagan JR (1991) Kinematics and control of frog hindlimb movements. J Neurophysiol 65:547-562.

Petersen PC, Vestergaard M, Jensen KH, Berg RW (2014) Premotor spinal network with balanced excitation and inhibition during motor patterns has high resilience to structural division. J Neurosci 34:2774-2784.

Pratt CA, Jordan LM (1987) Ia inhibitory interneurons and Renshaw cells as contributors to the spinal mechanisms of fictive locomotion. J Neurophysiol 57:56-71.

Radosevic M, Willumsen A, Petersen PC, Lindén H, Vestergaard M, Berg RW (2019) Decoupling of timescales reveals sparse convergent CPG network in the adult spinal cord. Nat Commun 10:2937.

Reali C, Russo RE (2013) Neuronal intrinsic properties shape naturally evoked sensory inputs in the dorsal horn of the spinal cord. Front Cell Neurosci 7:276.

Ritter DA, Bhatt DH, Fetcho JR (2001) In vivo imaging of zebrafish reveals differences in the spinal networks for escape and swimming movements. J Neurosci 21:8956-8965.

Robertson GA, Stein PS (1988) Synaptic control of hindlimb motoneurones during three forms of the fictive scratch reflex in the turtle. J Physiol 404:101-128.

Robertson GA, Mortin LI, Keifer J, Stein PS (1985) Three forms of the scratch reflex in the spinal turtle: central generation of motor patterns. J Neurophysiol 53:1517-1534.

Russo RE, Hounsgaard J (1994) Short-term plasticity in turtle dorsal horn neurons mediated by L-type $\mathrm{Ca}^{2+}$ channels. Neuroscience 61:191-197.

Russo RE, Nagy F, Hounsgaard J (1997) Modulation of plateau properties in dorsal horn neurones in a slice preparation of the turtle spinal cord. J Physiol 499:459-474.

Sandrini G, Serrao M, Rossi P, Romaniello A, Cruccu G, Willer JC (2005) The lower limb flexion reflex in humans. Prog Neurobiol 77:353-395.

Stein PS (2005) Neuronal control of turtle hindlimb motor rhythms. J Comp Physiol A Neuroethol Sens Neural Behav Physiol 191:213-229.

Stein PS (2008) Motor pattern deletions and modular organization of turtle spinal cord. Brain Res Rev 57:118-124.

Stein PS (2010) Alternation of agonists and antagonists during turtle hindlimb motor rhythms. Ann N Y Acad Sci 1198:105-118.

Stein PSG (2018) Central pattern generators in the turtle spinal cord: selection among the forms of motor behaviors. J Neurophysiol 119: 422-440.

Stein PSG, Robertson GA, Keifer J, Grossman ML, Berenbeim JA, Lennard PR (1982) Motor neuron synaptic potentials during fictive scratch reflex in turtle. J Comp Physiol 146:401-409.

Stein PS, McCullough ML, Currie SN (1998) Reconstruction of flexor/ex- 
tensor alternation during fictive rostral scratching by two-site stimulation in the spinal turtle with a transverse spinal hemisection. J Neurosci 18:467-479.

Stein PS, Camp AW, Robertson GA, Mortin LI (1986) Blends of rostral and caudal scratch reflex motor patterns elicited by simultaneous stimulation of two sites in the spinal turtle. J Neurosci 6:2259-2266.

Stein PS, Victor JC, Field EC, Currie SN (1995) Bilateral control of hindlimb scratching in the spinal turtle: contralateral spinal circuitry contributes to the normal ipsilateral motor pattern of fictive rostral scratching. J Neurosci 15:4343-4355.

Svirskis G, Hounsgaard J (1998) Transmitter regulation of plateau properties in turtle motoneurons. J Neurophysiol 79:45-50.
Triller A, Cluzeaud F, Pfeiffer F, Betz H, Korn H (1985) Distribution of glycine receptors at central synapses: an immunoelectron microscopy study. J Cell Biol 101:683-688.

Valk-Fai T, Crowe A (1978) Analyses of reflex movements in the hind limbs of the terrapin Pseudemys scripta elegans. J Comp Physiol 125:351-357.

Walløe S, Nissen UV, Berg RW, Hounsgaard J, Pakkenberg B (2011) Stereological estimate of the total number of neurons in spinal segment D9 of the red-eared turtle. J Neurosci 31:2431-2435.

Ziskind-Conhaim L, Hochman S (2017) Diversity of molecularly defined spinal interneurons engaged in mammalian locomotor pattern generation. J Neurophysiol 118:2956-2974. 